\title{
Water pair potential of near spectroscopic accuracy. I. Analysis of potential surface and virial coefficients
}

\author{
Eric M. Mas \\ Department of Physics and Astronomy, University of Delaware, Newark, Delaware 19716 \\ and Los Alamos National Laboratory, Los Alamos, New Mexico 87545 \\ Robert Bukowski \\ Department of Physics and Astronomy, University of Delaware, Newark, Delaware 19716 \\ and Department of Chemistry, Quantum Chemistry Laboratory, University of Warsaw, \\ Pasteura 1, 02-093, Warsaw, Poland \\ Krzysztof Szalewicz \\ Department of Physics and Astronomy, University of Delaware, Newark, Delaware 19716
}

Gerrit C. Groenenboom, Paul E. S. Wormer, and Ad van der Avoird

Institute of Theoretical Chemistry, NSR-Center, University of Nijmegen, Toernooiveld, 6525 ED Nijmegen, The Netherlands

(Received 5 June 2000; accepted 28 July 2000)

\begin{abstract}
A new $a b$ initio pair potential for water was generated by fitting 2510 interaction energies computed by the use of symmetry-adapted perturbation theory (SAPT). The new site-site functional form, named SAPT-5s, is simple enough to be applied in molecular simulations of condensed phases and at the same time reproduces the computed points with accuracy exceeding that of the elaborate SAPT-pp functional form used earlier [J. Chem. Phys. 107, 4207 (1997)]. SAPT-5s has been shown to quantitatively predict the water dimer spectra, see the following paper (paper II). It also gives the second virial coefficient in excellent agreement with experiment. Features of the water dimer potential energy surface have been analyzed using SAPT-5s. Average values of powers of the intermolecular separation-obtained from the ground-state rovibrational wave function computed in the SAPT-5s potential-have been combined with measured values to obtain a new empirical estimate of the equilibrium $\mathrm{O}-\mathrm{O}$ separation equal to $5.50 \pm 0.01$ bohr, significantly shorter than the previously accepted value. The residual errors in the SAPT-5s potential have been estimated by comparison to recent large-scale extrapolated ab initio calculations for water dimer. This estimatetogether with the dissociation energy $D_{0}$ computed from SAPT-5s-leads to a new prediction of the limit value of $D_{0}$ equal to $1165 \pm 54 \mathrm{~cm}^{-1}$, close to but significantly more accurate than the best empirical value. (C) 2000 American Institute of Physics. [S0021-9606(00)30140-4]
\end{abstract}

\section{INTRODUCTION}

The condensed phase of water is known for its interesting physical behavior. This is reflected by several anomalous properties, the best known of which are the density maximum at $4{ }^{\circ} \mathrm{C}$ and the fact that water at $0{ }^{\circ} \mathrm{C}$ and normal pressure is denser than ice. Thirty years of classical Monte Carlo (MC) and molecular dynamics (MD) simulations have provided much insight into the behavior of liquid water and ice. Yet, a quantitative statistical mechanical description which explains the anomalous properties is still lacking. There are good reasons to believe that this is mainly due to the insufficient knowledge of the intermolecular potential needed for the simulations. While the deviations of the many-body potential from pairwise additivity are substantial, knowledge of an accurate pair potential is the first step in achieving first-principles predictions of the properties of ice and liquid water. Most of the simulations to date used "effective" pair potentials: simple empirically parametrized model potentials in which the many-body interactions are represented implicitly. This is a drastic approximation and it is therefore not surprising that the results of the simulations with these model potentials are usually valid only for a re- stricted set of properties - often those to which the empirical parameters have been fitted-in a limited range of temperatures and pressures. Very recently Mahoney and Jorgensen ${ }^{1}$ obtained an empirical water pair potential, TIP5P, simultaneously fitting to $\mathrm{MC}$ simulations in a temperature range of $100{ }^{\circ} \mathrm{C}$. Although this range of applicability is significantly broader than for any other empirical potential to date, it nevertheless accentuates the limitations of the effective pair potentials. Simulations of more general validity will have to start from the real water pair potential, and explicitly include the important three-body interactions. This paper presents a new water pair potential computed by the use of symmetryadapted perturbation theory. Related work on three-body forces will be described elsewhere.

The first ab initio water pair potential was created by Matsuoka, Clementi, and Yoshimine (MCY) in $1976 .^{2}$ The MCY potential is a site-site form including Coulomb terms and double exponential terms fitted to 66 configuration interaction dimer energies. This form was quite successful at predicting observables despite the very small number of grid points and, from the current perspective, rather inaccurate values of ab initio computed energies. In fact, later increases 
by Clementi's group in the number of points and the level of theory led to potentials which predicted observables less satisfactorily. ${ }^{3,4}$ In the late 1980s Niessar, Corongiu, Clementi, and co-workers $(\mathrm{NCC})^{5,6}$ performed supermolecular fourth order many-body perturbation theory (MBPT4) level calculations (350 points) for the dimer and self-consistentfield (SCF) level calculations for the trimer (250 points). The former were fitted with a modified MCY form and the latter with the classical induction model. ${ }^{5,6}$ Another series of water potentials was developed by Stone and collaborators. ${ }^{7,8}$ The anisotropic site potential (ASP) of Millot and Stone ${ }^{7}$ models the electrostatic and induction effects by using explicitly calculated monomer properties (polarizabilities and multipole moments) in the form of an asymptotic expansion, the exchange effects by a fit to 350 points calculated with the Hayes-Stone intermolecular perturbation theory (IMPT), ${ }^{9}$ and the dispersion effects by an asymptotic damped expansion with constants calculated by Rijks and Wormer ${ }^{10}$ (ASP$\mathrm{W})$. The damping parameter in the Tang-Toennies function ${ }^{11}$ was assumed to be equal to the exponent modeling the exchange energy. Another version of the potential (ASP-S) used a fit to 33 ab initio computed dispersion energies by Szczęśniak et al. ${ }^{12}$ The ASP potential was modified by Millot et $\mathrm{al}^{8}{ }^{8}$ to produce the ASP-W2 and ASP-W4 potential energy surfaces (PESs).

Two fits to $a b$ initio water dimer potentials based on symmetry-adapted perturbation theory (SAPT) ${ }^{13-19}$ were obtained in Ref. 20. These fits utilized more grid points than any other previously published potential for water. The level of theory was approximately equivalent to MBPT4. A medium-size, interaction energy optimized basis set of $s p d f$ orbitals including bond functions was used. The accuracy of the interaction energy in the well region has been estimated at $0.2 \mathrm{kcal} / \mathrm{mol}$. One of the fits to the computed points in Ref. 20, dubbed SAPT-pp, is an expansion in functions of the Euler angles of the two monomers and the center of mass separation, while the other, less accurate SAPT-ss fit uses the MCY site-site form. The second virial coefficients calculated in Ref. 20 with the SAPT-pp/SAPT-ss PESs agreed with experimental values, including subtle effects of isotopic substitution, better than any prior ab initio prediction.

The present paper describes a new potential for the water dimer based on a larger number of grid points and a site-site functional form. The new potential, called SAPT-5s, has been used to calculate the water dimer and trimer spectra. ${ }^{21}$ A more detailed account of the calculations for the dimer can be found in Ref. 22, paper II. Many small tunneling splittings agree with experiment to within $0.01 \mathrm{~cm}^{-1}\left(3 \times 10^{-5} \mathrm{kcal} /\right.$ mol) while the larger splittings agree to within a few inverse centimeters $(\sim 0.01 \mathrm{kcal} / \mathrm{mol})$. This agreement is better than achieved by any other potential to date except for the very recent VRT(ASP-W) potential of Fellers et al. ${ }^{23}$ which, however, was fitted to these data. SAPT-5s was usedtogether with three-body nonadditive energies computed by us - in calculations of the spectra of the water trimer, again achieving near quantitative reproduction of the lowest energy levels. ${ }^{21}$ Replacement of SAPT-5s by the VRT(ASP-W) potential gave a substantial degradation in the quality of the trimer spectra. To further improve agreement with experi- ment, SAPT-5s has been empirically adapted to the water dimer spectrum, ${ }^{21}$ resulting in a "tuned" potential denoted by SAPT-5st. The present paper will discuss properties of both SAPT-5s and SAPT-5st surfaces (in particular the minimum geometry of the water dimer which can be compared to experiment) and the virial coefficients of water calculated with these potentials.

The plan of the paper is as follows. Section II describes how the additional grid points have been selected. The new functional form is proposed in Sec. III. SAPT-5s is compared to SAPT-pp in Sec. IV. In Sec. V the characteristic features of the SAPT-5s surface are analyzed. Section VI compares the virial coefficients computed using SAPT-5s with experiment. Section VII contains conclusions.

\section{SELECTION OF ADDITIONAL GRID POINTS}

The number of grid points used in the fit of the SAPT-pp potential was $1056 .^{20}$ In the late stages of this work, after the potentials described below were obtained, we found out that 53 of these points were overlapping, i.e., there were only 1003 distinct points. A double use of the same point amounts only to a stronger weighting of this point and should not have any significant effects on the fits. Therefore, the fits have not been repeated. In what follows we will refer to SAPT-pp as fitted to 1003 points. The SAPT-pp functional form of Ref. 20 was able to reproduce the calculated interaction energies quite well, the fit introducing errors similar to the estimated uncertainty of these energies. However, because of the complicated functional form, it is too timeconsuming to compute for applications to Monte Carlo or molecular dynamics simulations (although it can be used for calculations of dimer spectra and classical virial coefficients). Therefore, there was a need to produce a simpler potential of similar accuracy. Initial attempts to obtain a better than SAPT-ss site-site fit to 1003 ab initio points indicated that a denser grid is needed to achieve satisfactory results with this functional form. The original points were mostly on a regular grid. To make the coverage less dependent of arbitrary choices, 742 points have been chosen at random, however, with the range of the intermonomer center of mass distances, $R$, restricted to 4-6 bohr. To facilitate graphical analysis, for the next batch of 340 points only the angular configurations were chosen at random while $R$ was scanned between 4 and $7 \mathrm{bohr}$ with steps of $0.5 \mathrm{bohr}$. Analysis of the interaction energies from randomly generated grid points revealed that too many of those energies are in a highly repulsive region which is not relevant for the intended physical applications. Therefore, further grid points were added based on a Monte Carlo simulation of liquid water, calculations of the second virial coefficient, and in the region relevant for the dimer tunneling splitting dynamics. Overall, the original 1003 grid points were supplemented by 1507 new points. Calculations at all the additional grid points were done using the same basis set and level of theory as for the original 1003 points. However, for a number of grid points the bond functions were located slightly off the midpoint between the centers of mass, which introduced discrepancies of about $0.01-0.05 \mathrm{kcal} / \mathrm{mol}$ in interaction energies. Also, as 


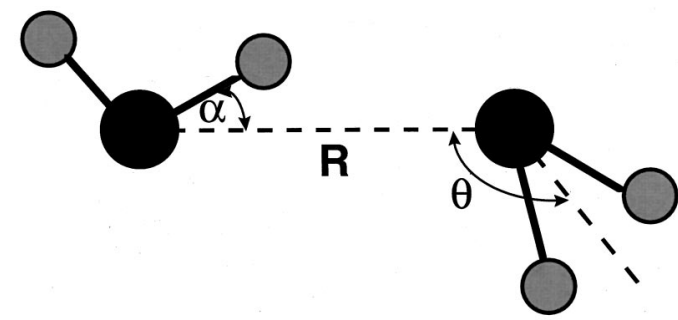

FIG. 1. Configuration of the dimer close to the minimum. $R$ is the distance between the centers of mass (COM) of the monomers and the angles are measured with respect to the line joining the two COMs.

in Ref. 20, the monomers were rigid at their average geometries of the ground-state vibration, as postulated in Ref. 24, see also Ref. 25.

The set of configurations obtained from a Monte Carlo simulation naturally does not include dimers with excessively repulsive energy. Since the long-range behavior of the potentials is believed to be already well represented in all SAPT fits (because of the use of independently calculated van der Waals coefficients, see below), only the intermediate- and short-range distances were considered. A short NVT Monte Carlo simulation with periodic boundary conditions was performed at the density of $1 \mathrm{~g} / \mathrm{cm}^{3}$ and temperature of $25{ }^{\circ} \mathrm{C}$ for 216 SAPT-pp water molecules. An initial configuration of a regular lattice of water molecules with random orientations was first equilibrated using the SAPT-ss PES. The SAPT-pp simulation was then equilibrated for only 20 or so cycles (a cycle is complete when a random move has been attempted on each of the $N$ molecules in the simulation). Another 10 cycles were run and all the pairs with separations less than 7 bohr were listed from the first and last cycle of the last phase of the run to produce a fairly large sample of configurations. Then 201 dimers were chosen randomly from this set.

Initial attempts to fit the original 1003 points with a site-site functional form-of flexibility high enough to achieve reproduction of computed energies with the same accuracy as that of SAPT-pp-were unsuccessful in the sense that the virial coefficient results were not satisfactory, particularly at high temperatures. Apparently the site-site fits achieved the accuracy of SAPT-pp at the computed points at the price of unphysical deformations in the regions not well covered by the grid. In order to identify configura-

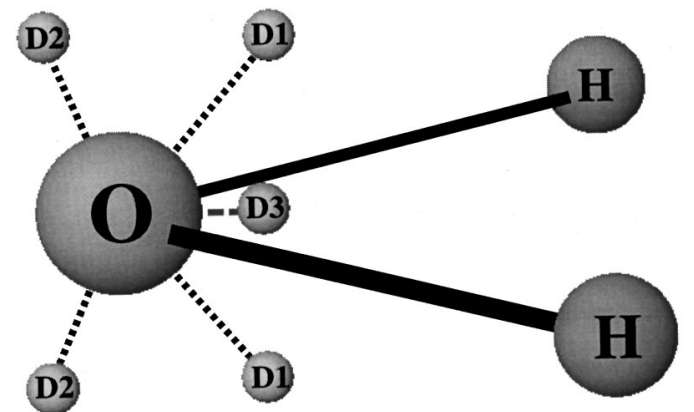

FIG. 2. Positions of sites in the SAPT-5s monomer.

tions responsible for the discrepancies in the second virial coefficient, this quantity was calculated simultaneously for the SAPT-pp and site-site potentials at $25^{\circ} \mathrm{C}$. At each integration point the contributions of the two potentials to the integral were compared and the 173 points which gave the largest differences were selected.

Additional 51 points were calculated in the dimer acceptor tunneling region. The tunneling pathway is shown in Fig. 1 of Ref. 22. First, 18 points were calculated for a linear hydrogen bond ( $\alpha=0$, cf. Fig. 1) with $R=5.62$ bohr. The acceptor angle ( $\theta$ in Fig. 1) took on the following five values: $122^{\circ}, 150^{\circ}, 180^{\circ}, 210^{\circ}$, and $238^{\circ}$. The angle associated with the torsion of the donor molecule around the axis joining the centers of mass $(\mathrm{COM})$ took on the values of $0^{\circ}$ (initial configuration), $30^{\circ}, 60^{\circ}$, and $90^{\circ}$ (two points among the 20 combinations of angles are redundant). An analogous scan was conducted for the nonlinear hydrogen bond with the angle $\alpha$ equal to $6.8^{\circ}$ (the donor torsion was still around the axis connecting COMs and the torsion angle was the dihedral angle defined by this axis and the donor planes before and after the rotation). Both scans were then repeated for $R=5$ bohr. The whole grid produces 72 points, however, a number of them were already included in the regular grid so that only 51 new points were computed. The final data set used in the fitting of the new pair potential contained SAPT interaction energies computed on a grid of 2510 dimer geometries. After the fit had been generated, we found that energies at two of the grid points were in error of about 0.3 $\mathrm{kcal} / \mathrm{mol}$ due to mistakes in input data. However, this had a negligible influence on the fit since its predictions for these

TABLE I. Geometry of the SAPT-5s pair potential and the roles the different sites play in the potential. Coordinates are given in bohr. A dot denotes that the site contributes to the given energy component. The site $D 3$ is at the center of mass computed here using values of 16 and 1 amu for the masses of oxygen and hydrogen, respectively.

\begin{tabular}{|c|c|c|c|c|c|c|}
\hline Site & $X$ & $Y$ & $Z$ & elst. & exp. & ind./disp. \\
\hline $\mathrm{O}$ & 0.0 & 0.0 & 0.1246319249 & - & - & - \\
\hline $\mathrm{H}$ & -1.4536519623 & 0.0 & -0.9970553993 & - & - & - \\
\hline $\mathrm{H}$ & 1.4536519623 & 0.0 & -0.9970553993 & - & - & - \\
\hline D1 & 0.0 & 0.2067213 & -0.247160511 & $\bullet$ & & \\
\hline D1 & 0.0 & -0.2067213 & -0.247160511 & $\bullet$ & & \\
\hline D2 & 0.0 & 0.20 & 0.25 & & - & \\
\hline D2 & 0.0 & -0.20 & 0.25 & & - & \\
\hline D3 & 0.0 & 0.0 & 0.0 & & $\bullet$ & \\
\hline
\end{tabular}


TABLE II. Cosine-type tesseral (Refs. 28 and 29) multipole moments $Q_{l m}$ calculated $a b$ initio, from the SAPT-5s charges (fitted to the $a b$ initio moments), from the SAPT-ss charges, and experimental values. All moments calculated in units of $e \cdot$ bohr $^{l}$ with respect to the center of mass of the water molecule located in the $x z$ plane with the $z$ axis coinciding with the molecular symmetry axis and the oxygen atom pointing in positive $z$ direction. The $\mathrm{O}-\mathrm{H}$ bond length is $1.8361063 \mathrm{bohr}$ and the $\mathrm{H}-\mathrm{O}-\mathrm{H}$ angle is 104.69 degrees.

\begin{tabular}{ccrrrr}
\hline \hline$l$ & $m$ & Ab initio & SAPT-5s & SAPT-ss & Expt. \\
\hline 1 & 0 & -0.75 & -0.75 & -0.73 & $-0.73^{\mathrm{a}}$ \\
2 & 0 & -0.12 & -0.12 & -0.21 & $-0.20^{\mathrm{b}}$ \\
2 & 2 & 2.17 & 2.12 & 2.08 & $2.20^{\mathrm{b}}$ \\
3 & 0 & 1.97 & 2.45 & 2.51 & \\
3 & 2 & -3.58 & -4.63 & -4.62 & \\
4 & 0 & -3.68 & -4.10 & -4.14 & \\
4 & 2 & 4.64 & 5.14 & 5.15 & \\
4 & 4 & 4.14 & 3.72 & 3.74 & \\
5 & 0 & 0.84 & 1.28 & 1.30 & \\
5 & 2 & 1.48 & 0.76 & 0.76 & \\
5 & 4 & -12.42 & -11.14 & -11.19 & \\
6 & 0 & 9.89 & 8.28 & 8.31 & \\
6 & 2 & -16.94 & -14.39 & -14.45 & \\
6 & 4 & 22.77 & 19.56 & 19.64 & \\
6 & 6 & 9.41 & 7.15 & 7.18 & \\
7 & 0 & -22.17 & -18.76 & -18.83 & \\
7 & 2 & 30.29 & 25.82 & 25.93 & \\
7 & 4 & -19.42 & -17.18 & -17.26 & \\
7 & 6 & -34.24 & -25.70 & -25.81 & \\
8 & 0 & 12.98 & 12.55 & 12.60 & \\
8 & 2 & -9.87 & -11.12 & -11.17 & \\
8 & 4 & -20.99 & -13.30 & -13.36 & \\
8 & 6 & 77.11 & 57.51 & 57.74 & \\
8 & 8 & 20.98 & 14.10 & 14.15 & \\
\hline \hline
\end{tabular}

${ }^{\mathrm{a}}$ Reference 30.

${ }^{\mathrm{b}}$ Reference 31 .

points agreed to $0.04 \mathrm{kcal} / \mathrm{mol}$ with the correct interaction energies.

\section{SAPT-5s FIT}

Applying prior experience, a new site-site pair potential, SAPT-5s, was fitted to the 2510 dimer energies. The " 5 ", and "s" stand for 5 (symmetrically distinct) sites on each monomer. The positions of the sites along with the roles they play for the new potential are provided in Table I and illustrated in Fig. 2. The new functional form is

$$
\begin{aligned}
E_{\mathrm{int}}= & \sum_{a \in A, b \in B}\left[f_{1}\left(\delta_{1}^{a b}, R_{a b}\right) \frac{q_{a} q_{b}}{R_{a b}}+g^{a b}\left(R_{a b}\right) \mathrm{e}^{\alpha_{a b}-\beta_{a b} R_{a b}}\right. \\
& \left.+\sum_{n=6,8,10} f_{n}\left(\delta_{n}^{a b}, R_{a b}\right) \frac{C_{n}^{a b}}{\left(R_{a b}\right)^{n}}\right]
\end{aligned}
$$

where the sum extends over all sites $a(b)$ in molecule $A(B)$. The first term represents the Coulombic interactions between sites. As is commonly done in angular expansions such as SAPT-pp, but not often in site-site forms, this term has been damped using the Tang-Toennies function, ${ }^{11}$

$$
f_{n}(\delta, R)=1-e^{-\delta R} \sum_{m=0}^{n}(\delta R)^{m} / m \text { ! }
$$
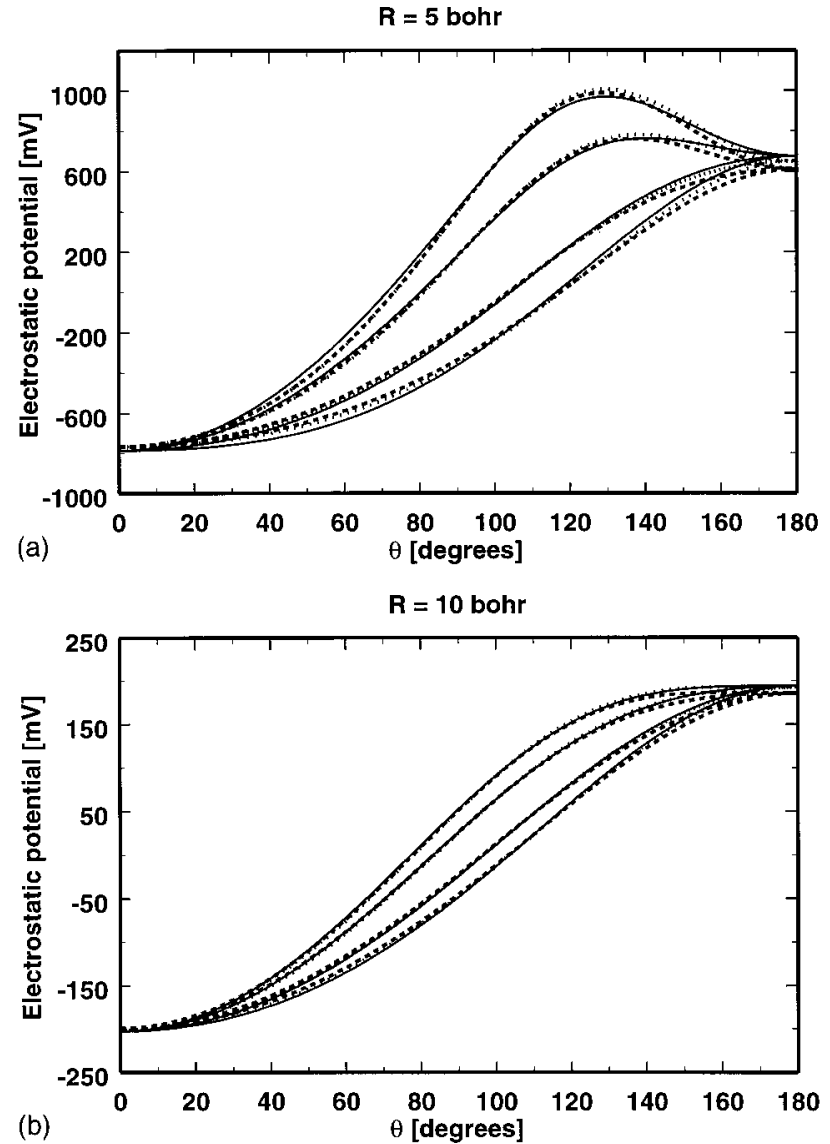

FIG. 3. Electrostatic potential around a water molecule at 5 and $10 \mathrm{bohr}$. Compared are calculations conducted with SAPT-ss charges (dashed line), SAPT-5s charges (dotted line), and the multipole expansion (solid line). The angles $\theta$ and $\phi$ are polar angles of a point in space around a water molecule located in the $x-z$ plane with its center of mass at the origin of the coordinate system and with the oxygen on the positive $z$ axis. From top to bottom $\phi=0^{\circ}, 30^{\circ}, 60^{\circ}$, and $90^{\circ}$. The potential is in millivolts.

Damping is particularly important for site-site models where the sites can get much closer than the center of mass separation used in the angular expansion while the dimer is still in an energetically important region.

The three distinct charges (at five positions) were determined by a least-squares fit to the center of mass monomer multipole moments. These moments were computed $a b$ initio at the MBPT3 level with orbital relaxation using the POLCOR program of Wormer and Hettema ${ }^{26,27}$ and the finitefield method. The monomer-centered part of the basis set used in the dimer calculations was employed. A weighting factor of $10^{-3 l-k}$ (where $l$ and $k$ enumerate the multipole moment components connected with spherical harmonics $Y_{l k}$, or more precisely with their tesseral form) assured that the dipole and quadrupole moments were reproduced with very high accuracy, while confining the higher moments to reasonable values. The highest $l$ value was equal to 8 . The total charge was enforced to be zero. The positions of the off-atomic sites, $D 1$, constrained to preserve the $\mathrm{C}_{2 v}$ symmetry of the monomer, were optimized during the fitting process.

The $a b$ initio calculated multipole moments are compared with values resulting from the fitted charges and experimental data ${ }^{30,31}$ in Table II. Also quantities resulting 
TABLE III. SAPT-5s charges.

\begin{tabular}{cc}
\hline \hline Site & \multicolumn{1}{c}{ Charge } \\
\hline $\mathrm{O}$ & 0.2585046 \\
$\mathrm{H}$ & 0.5640504 \\
$\mathrm{D} 1$ & -0.6933027 \\
\hline
\end{tabular}

from SAPT-ss ${ }^{20}$ charges are given. Notice that although SAPT-ss is not a very accurate fit, its charges-which were free parameters of the dimer fit-give the three lowest multipole moments which agree with experimental values very well. While the higher multipole moments computed from the SAPT-5s fitted charges seem to be significantly different from the $a b$ initio directly computed values, it is important to note that these high moments make rather small contributions to the electrostatic potential of a molecule. This potential is shown in Fig. 3 as defined by SAPT-5s charges, SAPT-ss charges, and the multipole expansion using the multipoles from the SAPT-pp potential at 5 and $10 \mathrm{bohr}$. At both distances, even at the very short separation of $5 \mathrm{bohr}$, the electrostatic potentials produced by the SAPT-5s and SAPT-ss charges agree very well with the multipole expansion.

The second term in Eq. (1) represents the exponentially decaying portion of the potential. The polynomial

$$
g(R)=\kappa\left(1+\sum_{m=1}^{3} a_{m} R^{m}\right),
$$

where $\kappa$ is an appropriate energy unit, has the effect of increasing the flexibility of the fit. It is physically motivated by the fact that exponential contributions are not limited to the repulsive exchange energy, and some of them may be attractive. Incorporation of the polynomial represents the sum of all such contributions more closely than a single exponential term. The positions of the $D 2$ and $D 3$ sites utilized by the exponential term were determined by rough optimizations.

The third term in Eq. (1) represents the induction and dispersion energies. The $C_{6}^{a b}, C_{8}^{a b}$, and $C_{10}^{a b}$ terms were least-squares fitted to the asymptotic induction and dispersion energies calculated-using the center of mass van der Waals coefficients - on a grid of 2510 points with the same angular orientations as the SAPT energy data set, but with $R$ incremented by $3 \AA$. The center of mass coefficients were obtained using the POLCOR programs ${ }^{26,27}$ applying the level of theory and basis set compatible with the SAPT calculations. The induction-dispersion sites were placed only on the atomic nuclei. The unweighted standard deviation per point of this fit was $0.002 \mathrm{kcal} / \mathrm{mol}$ for the 2510 points. Thus, the discrepancies between the fit and the original expansion are too small to be visible in a figure.

TABLE IV. SAPT-5s induction/dispersion coefficients. Units are (kcal/mol) $\AA^{n}, n=6,8,10$.

\begin{tabular}{rcrrr}
\hline \hline $\mathrm{a}$ & $\mathrm{b}$ & \multicolumn{1}{c}{$-C_{6}$} & \multicolumn{1}{c}{$-C_{8}$} & \multicolumn{1}{c}{$-C_{10}$} \\
\hline $\mathrm{O}$ & $\mathrm{O}$ & -418.9423520 & 38830.4051653 & -462206.9340918 \\
$\mathrm{H}$ & $\mathrm{O}$ & 410.6137764 & -12799.7555426 & 145609.6937164 \\
$\mathrm{H}$ & $\mathrm{H}$ & -41.9833633 & 1069.1814818 & -4294.7493548 \\
\hline \hline
\end{tabular}

TABLE V. SAPT-5s electrostatic damping parameters.

\begin{tabular}{lcc}
\hline \hline $\mathrm{a}$ & $\mathrm{b}$ & $\delta_{1}$ \\
\hline $\mathrm{O}$ & $\mathrm{O}$ & 5.3018840 \\
$\mathrm{H}$ & $\mathrm{O}$ & 1.4026462 \\
$\mathrm{H}$ & $\mathrm{H}$ & 1.3777702 \\
$\mathrm{D} 1$ & $\mathrm{O}$ & 4.8825786 \\
$\mathrm{D} 1$ & $\mathrm{H}$ & 1.4506083 \\
$\mathrm{D} 1$ & $\mathrm{D} 1$ & 1.6247997 \\
\hline \hline
\end{tabular}

Once the charges, $D i$ site positions, and $C_{6}^{a b}, C_{8}^{a b}$, and $C_{10}^{a b}$ parameters were established, they were kept constant, i.e., they were not free parameters of the overall fit. This constrained these long-range electrostatic, induction, and dispersion terms to reproduce the asymptotic energies they were intended for, just like the explicitly calculated van der Waals coefficients did for the SAPT-pp PES. We have found that this was critical for getting a reasonably behaving fit. Attempts at unrestricted optimizations ended up with fits which reproduced the computed points very well, but gave unreasonable predictions of observables and independent check points.

Finally, the damping parameters $\left(\delta_{n}^{a b}\right)$, exponents $\left(\alpha_{a b}\right.$, $\left.\beta_{a b}\right)$, and coefficients of the polynomial multiplying the exponential term $\left(a_{m}\right), 65$ free parameters in total, were fitted to the set of 2510 data points. The weighting function

$$
w=\left(\frac{1}{E+5.5 \mathrm{kcal} / \mathrm{mol}}\right)^{2}
$$

was used to ensure a good fit to the low-energy configurations. The parameters of the SAPT-5s PES are provided in Tables III-VIII and a subroutine to calculate the interaction energies is available. ${ }^{32}$

Table IX presents the unweighted standard deviations per point for various fits. The deviations computed including all grid points do not provide the complete picture since such deviations are dominated by contributions from points high on the repulsive wall, less relevant for physical applications. Therefore we also give in Table IX deviations for points with energies less than $10 \mathrm{kcal} / \mathrm{mol}$ and with negative energies. As this table shows, the unweighted standard deviation per point is only $0.16 \mathrm{kcal} / \mathrm{mol}$ for the most relevant region with energies less than $10 \mathrm{kcal} / \mathrm{mol}$, i.e., it is smaller than the estimated uncertainties of the $a b$ initio points (about $0.3 \mathrm{kcal} /$ mol near the minimum) showing that the accuracy of SAPT-5s is very satisfactory.

To investigate the accuracy of the SAPT- 5 s potential in regions not represented by the 2510 data points used in its generation, additional 50 points were computed by searching for "holes" in the 2510 point data set. In order to determine where the holes were, a criteria describing the "distance",

TABLE VI. SAPT-5s induction/dispersion damping parameters.

\begin{tabular}{ccccc}
\hline \hline $\mathrm{a}$ & $\mathrm{b}$ & $\delta_{6}$ & $\delta_{8}$ & $\delta_{10}$ \\
\hline $\mathrm{O}$ & $\mathrm{O}$ & 1.2817400 & 2.1167487 & 6.2405802 \\
$\mathrm{H}$ & $\mathrm{O}$ & 9.3716776 & 1.0359837 & 3.0409734 \\
$\mathrm{H}$ & $\mathrm{H}$ & 5.8292032 & 4.9506181 & 3.5438175 \\
\hline \hline
\end{tabular}


TABLE VII. SAPT-5s exponential parameters.

\begin{tabular}{llrr}
\hline \hline Site a & $\mathrm{b}$ & \multicolumn{1}{c}{$\alpha$} & \multicolumn{1}{c}{$\beta$} \\
\hline $\mathrm{O}$ & $\mathrm{O}$ & 14.6457300 & 3.9653333 \\
$\mathrm{H}$ & $\mathrm{O}$ & 7.5142110 & 3.3134498 \\
$\mathrm{H}$ & $\mathrm{H}$ & 7.3488991 & 2.4142921 \\
$\mathrm{D} 2$ & $\mathrm{O}$ & 11.2060117 & 3.9634120 \\
$\mathrm{D} 2$ & $\mathrm{H}$ & 9.7649989 & 3.4217174 \\
$\mathrm{D} 2$ & $\mathrm{D} 2$ & 5.5646260 & 3.9611226 \\
D3 & $\mathrm{O}$ & 6.5618764 & 3.9767526 \\
D3 & $\mathrm{H}$ & 4.9867889 & 3.1725653 \\
D3 & $\mathrm{D} 2$ & 6.5971413 & 3.9680667 \\
D3 & $\mathrm{D} 3$ & 3.6913373 & 3.9871502 \\
\hline \hline
\end{tabular}

between configurations needed to be established. Instead of describing dimers by $R$ and Euler angles, we characterized them by the nine intermolecular atom-atom separations, $R_{\mathrm{O} 1-\mathrm{O} 2}, \quad R_{\mathrm{O} 1-\mathrm{H} 3}, R_{\mathrm{O} 1-\mathrm{H} 4}, R_{\mathrm{O} 2-\mathrm{H} 1}, R_{\mathrm{O} 2-\mathrm{H} 2}, R_{\mathrm{H} 1-\mathrm{H} 3}$, $R_{\mathrm{H} 1-\mathrm{H} 4}, R_{\mathrm{H} 2-\mathrm{H} 3}$, and $R_{\mathrm{H} 2-\mathrm{H} 4}$ where $\mathrm{O} 1, \mathrm{H} 1$, and $\mathrm{H} 2$ are the atomic sites on one molecule and $\mathrm{O} 2, \mathrm{H} 3$, and $\mathrm{H} 4$ on the other. The four oxygen-hydrogen intermolecular $R_{\mathrm{O} i-\mathrm{H} j}$ distances were arranged in ascending order, as were the four $R_{\mathrm{Hi}-\mathrm{H} j}$ distances. We generated 100000 random dimers with center of mass separations between 4.5 and 7 bohr, placed in bins of 0.5 bohr length. The "distance" $D$ between one of the 100,000 random dimers and one of the 2510 grid dimers, characterized by the sorted distances vector $R_{X Y}^{\prime}$, was computed as

$$
D=\sqrt{\sum_{1}^{9}\left(R_{X Y}-R_{X Y}^{\prime}\right)^{2}} .
$$

For each random dimer the smallest $D$, denoted by $D_{\min }$, defines the "distance" between this dimer and the 2510 point set. The 10 points with the largest $D_{\min }$ in each of the five $R$ bins were taken as the 50 geometries least well represented in the 2510 point data set. SAPT runs were carried out for these 50 points and the calculated energies were compared with the values predicted by the fit. The unweighted standard deviation per point was $0.42 \mathrm{kcal} / \mathrm{mol}$ for all 50 points, $0.20 \mathrm{kcal} / \mathrm{mol}$ for points with energies less than 10 $\mathrm{kcal} / \mathrm{mol}$, and $0.02 \mathrm{kcal} / \mathrm{mol}$ for points with negative energies, which are similar to the errors in points used in the fit. The quality of the SAPT-5s PES is also illustrated in Fig. 4 where the interaction energies are plotted versus the 2510 fit values (circles) and the 50 additional points not used in the fit (triangles). The virtual indistinguishability of the range of scatters indicates that SAPT-5s predicts dimer energies for geometries not used in the fit equally well as it does for those used in the fit. Both the plot and the standard deviations show that SAPT-5s should be an adequate approximation of the $a b$ initio interaction energy in regions not well represented by the 2510 grid points.

Since the parts of the potential function containing inverse powers of $R$ tend to minus infinity as $R$ approaches zero while the exponential terms tend to a constant, the potentials become unphysical for very small $R$. This behavior happens for values of $R$ which are irrelevant for virtually all physical problems. Nevertheless, some of our previous potentials have been regularized at small $R .{ }^{33}$ We checked that SAPT-5s shows unphysical behavior not earlier than after reaching about $60 \mathrm{kcal} / \mathrm{mol}$ on the repulsive wall. Therefore, regularization was not needed.

\section{COMPARISON OF SAPT-5s WITH SAPT-pp}

Table IX shows that the fit quality of SAPT-5s on the 1003 original points is somewhat better than the quality of SAPT-pp on the same points, despite the fact that SAPT-5s was fitted to the larger set of 2510 points. This indicates that site-site fits can reach accuracy comparable to more sophisticated angular expansion fits. One should point out that this is achieved with a smaller number of free parameters. There were 65 such parameters used in SAPT-5s, while each of the five separately fitted interaction energy component contributing to SAPT-pp used 106 or 107 free parameters. ${ }^{20}$ Even more importantly, SAPT-5s is much less time consuming to compute, in fact, by as much as 1400 times less.

High accuracy in reproducing fitted points may be achieved at the cost of poor predictions in the regions less densely covered by the grid. As discussed in the preceding section, this is not the case for SAPT-5s. However, based on our experience from the early stages of this work we suspected that the SAPT-pp form is better than SAPT-5s in predicting new points. To check this we obtained the SAPT-5s form fit to the original 1003 points only and this fit will be denoted by SAPT-5s/1003. As can be seen in Table IX, the unweighted standard deviations of this fit on the set of 1003 points are virtually the same as those of SAPT-5s and therefore are somewhat better than those produced by SAPT-pp. However, when the SAPT-5s/1003 fit was applied to predict the energies for the 2510 point data set, the stan-

TABLE VIII. SAPT-5s coefficients of polynomial multiplying the exponential term.

\begin{tabular}{llrrr}
\hline \hline Site a & $\mathrm{b}$ & \multicolumn{1}{c}{$a_{1}$} & \multicolumn{1}{c}{$a_{2}$} & \multicolumn{1}{c}{$a_{3}$} \\
\hline $\mathrm{O}$ & $\mathrm{O}$ & 42.4349609 & -57.9143175 & 21.8222941 \\
$\mathrm{O}$ & $\mathrm{H}$ & 55.3348022 & -49.0389806 & 28.9395322 \\
$\mathrm{O}$ & $\mathrm{D} 2$ & -345.7441865 & 458.8277255 & -170.0811798 \\
$\mathrm{O}$ & $\mathrm{D} 3$ & -75803.0380142 & 100152.0266287 & -37910.8764418 \\
$\mathrm{H}$ & $\mathrm{H}$ & -0.3335947 & -0.02084309 & 0.06740784 \\
$\mathrm{H}$ & $\mathrm{D} 2$ & -3.3142387 & 2.6259084 & -1.1633726 \\
$\mathrm{H}$ & $\mathrm{D} 3$ & -220.4414512 & 152.6105586 & -114.2353923 \\
$\mathrm{D} 2$ & $\mathrm{D} 2$ & 23840.7041001 & -31957.5522798 & 11806.6845163 \\
D2 & D3 & 17574.6806682 & -23397.6987314 & 8852.7868527 \\
D3 & D3 & 698033.8268711 & -921648.6319947 & 354509.6357180 \\
\hline \hline
\end{tabular}


TABLE IX. Unweighted standard deviation per point (in $\mathrm{kcal} / \mathrm{mol}$ ) for SAPT-pp, SAPT-5s, and SAPT-5s fitted to 1003 points only. The consecutive pairs of columns are for the total set of 1003 (2510) data points, its subset of 984 (2083) points whose energies are less than $10 \mathrm{kcal} / \mathrm{mol}$, and the 467 (1045) points with negative energies.

\begin{tabular}{|c|c|c|c|c|c|c|}
\hline & \multicolumn{2}{|c|}{ All } & \multicolumn{2}{|c|}{$E_{\text {int }}<10$} & \multicolumn{2}{|c|}{$E_{\text {int }}<0$} \\
\hline & 1003 & 2510 & 1003 & 2510 & 1003 & 2510 \\
\hline SAPT-pp & 0.31 & 2.5 & 0.20 & 0.49 & 0.10 & 0.33 \\
\hline SAPT-5s & 0.16 & 0.37 & 0.15 & 0.18 & 0.09 & 0.10 \\
\hline SAPT-5s/1003 & 0.14 & 12.2 & 0.12 & 0.56 & 0.07 & 0.10 \\
\hline
\end{tabular}

${ }^{a}$ The SAPT-5s form fit to the 1003 point set used in Ref. 20.

dard deviations were larger than those given by SAPT-pp, except in the region where the potential is negative. The latter effect is probably due to the very strong weighting of the well region by Eq. (4) in comparison to the weights used in fitting SAPT-pp. The finding that SAPT-5s/1003 performs worse than SAPT-pp in predicting the interaction energies for points not used in the fitting process explains our initial difficulties with flexible site-site fit forms and confirms the necessity of the increase of the number of grid points.

One may want to find reasons why the simpler SAPT-5s form is able to surpass the accuracy of the SAPT-pp form, while at the same time predictions for the points not used in the fit seem to be more robust for SAPT-pp. We believe that the main advantages of SAPT-5s come from its distributed character which is more suitable for modeling the features of the potential energy surface related to the shapes of molecules. The better predictive power of SAPT-pp is probably due to the fact that SAPT-pp was fitted component-bycomponent, while SAPT-5s was fitted to the total interaction energy. Each component exhibits a much more monotonic dependence on the geometry of the dimer than the total potential, therefore it is simpler to fit. Also the weights used in fitting SAPT-pp were adjusted to each component and were different than given by Eq. (4), probably better emphasizing the importance of various regions of configuration space. One could use, of course, the SAPT-5s functional form to fit individual interaction energy components, but in view of excellent performance of the current SAPT-5s fit this does not appear necessary.

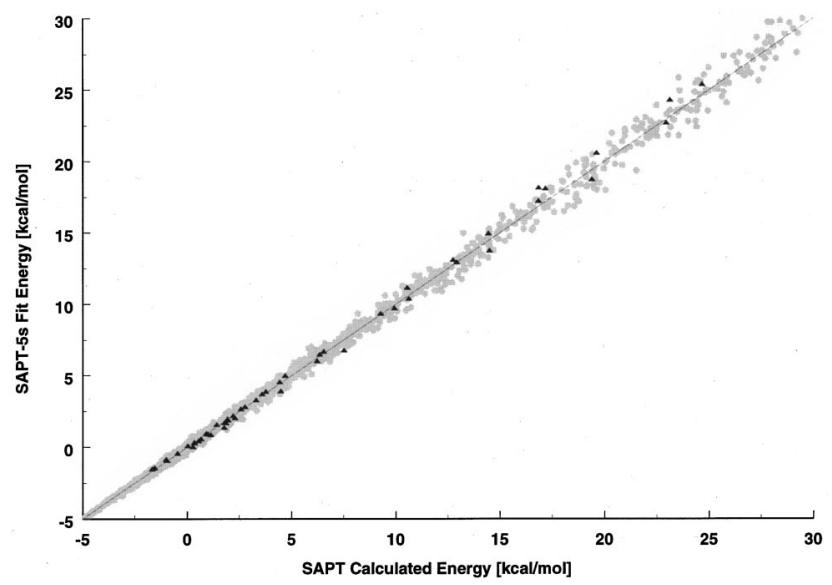

FIG. 4. Quality of the SAPT-5s fit. Circles represent the 2510 points used in the fit and triangles represent additional points not used in the fit.

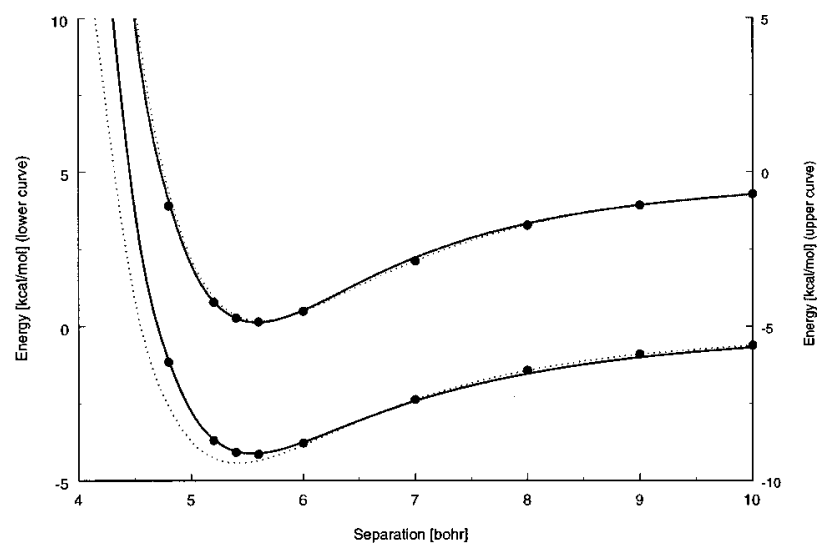

FIG. 5. Comparison of the SAPT-pp (dotted line) and SAPT-5s (solid line) potentials along two radial scans with SAPT $a b$ initio energies shown as large dots. None of these points were used in either fit. The upper scan passes through the dimer minimum and energies can be read off of the right $y$ axis. The lower scan passes through a dimer which corresponds to the minimum energy cyclic planar trimer.

Let us now directly compare the major differences between SAPT-5s and SAPT-pp potentials in various regions. The largest such differences proved to be at short range. For geometries other than the minimum orientation, the SAPT-pp potential produces dimer energies which are too negative inside the well for distances shorter than the minimum energy separation. Figure 5 illustrates this problem by showing two radial cuts through the SAPT-pp and SAPT-5s PESs. The top scan passes through the dimer minimum geometry as defined by SAPT-5s $\left(\alpha=6.3^{\circ}\right.$ and $\theta=127^{\circ}$, from Fig. 1) and the lower scan passes through the configuration of a dimer in the trimer cyclic planar structure with the hydrogen bonds deviating 21.2 $2^{\circ}$ from linearity (COM$\mathrm{COM}-\mathrm{H}$ angle). None of the ab initio points shown in Fig. 5 have been used in either fit, therefore the figure demonstrates well the better predictive power of SAPT-5s. On the upper curve, going through the global minimum, SAPT-pp performs equally well as SAPT-5s. The reason is that this curve passes very near the region well covered in the original 1003 point set. In contrast, this set contained few points in the region related to the trimer configurations. In general, SAPT-pp is a very good approximation to the computed points in the region well represented by the original set of 1003 data points. In the trimer configuration region, which was not well sampled by this set, the SAPT-pp curve is too deep at separations shorter than $R_{\min }$. In contrast, SAPT-5s provides an excellent approximation to the computed points in both regions. An insufficient number of original points lying in the well inside the minimum separation is itself a cause for the discussed behavior of SAPT-pp. The other reason is that the angular expansion centered on the centers of mass may have troubles dealing with charge overlap effects experienced by atomic sites which get close to each other while $R$ is still large. In such cases, the site-site exponential terms may represent the physical effects of overlap better than the single-center angular expansion.

Further comparisons of results obtained with the SAPT-pp and SAPT-5s potentials will be presented later in this paper. It will be seen that SAPT-5s reproduces the $a b$ 
TABLE X. Dimer equilibrium properties predicted by SAPT-5s. Angle $\alpha$ is the deviation of the hydrogen bond from linearity relative to the oxygen-oxygen axis and $\theta$ is the angle between the line connecting the oxygens and the symmetry axis of the hydrogen acceptor. Notice that in other places in this paper the same symbols denote related angles determined with respect to the centers of mass of the monomers. Energies correspond to the geometries listed in a given column except for the last two columns. All distances are in bohr, angles in degrees, and energies in $\mathrm{kcal} / \mathrm{mol}$.

\begin{tabular}{lcccccrr}
\hline \hline & SAPT $^{\mathrm{a}}$ & SAPT-pp & SAPT-5s & CCSD(T)/MBPT2 & CCSD(T)/MP2-R12 & Extrapolated $^{\mathrm{b}}$ & Expt. $^{2}$ \\
\hline$\alpha_{e}$ & 4.6 & 1.0 & 6.3 & $4.3^{\mathrm{c}}$ & $4.8^{\mathrm{d}}$ & 5.5 & $5 \pm 10^{\mathrm{e}}$ \\
$\theta_{e}$ & 124 & 149 & 127 & $128.2^{\mathrm{c}}$ & $122.5^{\mathrm{d}}$ & 124.4 & $123 \pm 10^{\mathrm{e}}$ \\
$R_{\mathrm{OO}, e}$ & 5.58 & 5.566 & 5.584 & $5.527^{\mathrm{c}}$ & $5.472^{\mathrm{d}}$ & $5.503 \pm 0.01$ & $5.50 \pm 0.01^{\mathrm{f}}$ \\
$E_{\min }$ & -4.86 & -4.91 & -4.86 & $-4.92^{\mathrm{g}}$ & $-4.94^{\mathrm{h}}$ & $-5.02 \pm 0.05$ & $-5.44 \pm 0.7^{\mathrm{i}}$ \\
\hline \hline
\end{tabular}

${ }^{\mathrm{a}} \mathrm{A} b$ initio values.

${ }^{\mathrm{b}}$ Extrapolated results of Klopper et al. (Ref. 38), see text.

${ }^{c}$ Frozen-core, CP-corrected CCSD(T) optimization by Schütz et al. (Ref. 34) in a 148 term ANO basis. Monomers were rigid at their geometries in a dimer from a RASSCF optimization.

${ }^{\mathrm{d}}$ Frozen-core, CP-uncorrected CCSD(T)/aug-cc-pVTZ optimization by Halkier et al. (Ref. 35) with monomers frozen at the experimental equilibrium geometry.

${ }^{\mathrm{e}}$ Reference 39.

${ }^{\mathrm{f}}$ Experimental $\left\langle R_{\mathrm{OO}}^{-2}\right\rangle^{-1 / 2}=5.624 \mathrm{bohr}$ from Ref. 39 plus the anharmonicity correction from the present work.

${ }^{\mathrm{g}}$ All-electrons, CP-corrected MBPT2 result (relative to deformed monomers) of Schütz et al. (Ref. 34) in a 1046 term basis.

${ }^{\mathrm{h}}$ All-electrons, CP-corrected MP2-R12 result of Klopper and Lüthi, Ref. 36.

${ }^{\mathrm{i}}$ Reference 40 . A revised value obtained in the present work is $5.00 \pm 0.7 \mathrm{kcal} / \mathrm{mol}$.

initio minimum geometry better than SAPT-pp which is clearly related to the weighting differences discussed above. At low temperatures the SAPT-pp/ss combination gives somewhat better second virial coefficients than SAPT-5s does but we find this to be an artifact given by the SAPT-ss fit. Also the dimer spectra have been computed with SAPTpp. While some features of the spectra like dissociation energies, rotational constants, and the acceptor splittings are very similar between the two potentials (differences smaller than the deviations of either spectrum from experiment), the small interchange splittings, which SAPT-5s recovers to within a few percent, deviate from experiment on the order of $20 \%$ in the case of the SAPT-pp potential. Still, SAPT-pp splittings are better than those of any other published potential except for the VRT(ASP-W) which was empirically fitted to reproduce these splittings. ${ }^{23}$ The improvement of the interchange splittings brought by the SAPT-5s potential is clearly due to the increased number of grid points and better reproduction of the well region (cf. the last column of Table IX).

\section{ANALYSIS OF SAPT-5S POTENTIAL ENERGY SURFACE}

\section{A. Minimum}

\section{Geometry}

The minimum energy and configuration predicted by the SAPT-5s potential are compared in Table $\mathrm{X}$ with experimental values and recent large-scale calculations for the water dimer. ${ }^{34-38}$ One should notice that the supermolecular calculations discussed below use monomers at their equilibrium, " $r_{e}$ ", geometry and in some cases search for the total minimum on the dimer surface including a variation of monomer internal coordinates, while SAPT calculations use the ground-state vibrationally averaged, " $\langle r\rangle_{0}$ ", geometry (sometimes also denoted by $r_{0}$ ) rigid monomers. Table $\mathrm{X}$ shows that the SAPT-5s dimer minimum parameters are very close to the SAPT values computed $a b$ initio $^{24}$ showing again the excellent quality of the fit. All the calculations listed in Table X, except for SAPT-pp, predict the angular orientation of the water monomers to within experimental ${ }^{39}$ error limits. (Notice that in Table X for the purpose of comparison with literature the angles are measured with respect to the line connecting the oxygens rather than the centers of mass of each monomer.) The situation is quite different for the equilibrium minimum $R_{\mathrm{OO}}$ separation and in fact this issue has been somewhat of a controversial subject in recent years. Results from various calculations spanned a wide range of values. Even the two most advanced minimizations, by Schütz et al. ${ }^{34}$ and Halkier et al. ${ }^{35}$-performed using the coupled cluster method with single, double, and noniterative triple excitations $[\mathrm{CCSD}(\mathrm{T})]$ and medium-size $s p d f$ basis sets of comparable quality - differ by as much as 0.06 bohr. In this case the discrepancy can be related to the fact that the former result was corrected for the basis set superposition error (BSSE) with the counterpoise (CP) correction of Boys and Bernardi ${ }^{41}$ while the latter was not. Moreover, most of the calculations produced $R_{\mathrm{OO}, e}$ much shorter (of the order of $0.1 \mathrm{bohr}$ ) than the generally accepted "experimental" value of the equilibrium $R_{\mathrm{OO}, e}$ separation equal to $5.578 \mathrm{bohr}$. This value was inferred by Odutola and Dyke ${ }^{39}$ from the measured rotational constants. First, using a rigid monomer model they deduced $\left\langle R_{\mathrm{OO}}^{-2}\right\rangle^{-1 / 2}=5.624 \pm 0.008 \mathrm{bohr}$. Then, using a diatomic molecule model they estimated that the effect of anharmonicity leads to $R_{\mathrm{OO}, e} 0.045 \mathrm{bohr}$ shorter than $\left\langle R_{\mathrm{OO}}^{-2}\right\rangle^{-1 / 2}$, giving the equilibrium separation quoted above. Notice that the error bars of this value are unknown and are certainly larger than 0.008 bohr. Recently, Klopper, van Duijneveldt-van de Rijdt, and van Duijneveldt ${ }^{38}$ performed an elaborate search for the minimum geometry using largescale MBPT2 through $\operatorname{CCSD}(\mathrm{T})$ calculations with interaction-optimized orbital basis sets as well as with explicitly correlated bases and extrapolation techniques. Their final estimate of $5.503 \pm 0.01 \mathrm{bohr}$ is most likely the definitive result, however, it is rather far from the value deduced from experiment. ${ }^{39}$

Our calculations described in paper $\mathrm{II}^{22}$ bring a resolution to this issue. The value of $\left\langle R_{\mathrm{OO}}^{-2}\right\rangle^{-1 / 2}$ computed on the 
SAPT-5s potential is $5.706 \mathrm{bohr}$ for the ground state, $J=K$ $=0$, and 5.701 bohr for the $J=1, K=0$ state. Since the rotational constants are obtained both in experiment ${ }^{39}$ and in theory $^{22}$ from the difference of the rotational energies of these two states, we take the average of the two distances. Since $R_{\mathrm{OO}, e}=5.584$ bohr for SAPT-5s, this gives $\Delta R_{\mathrm{OO}}$ $=\left\langle R_{\mathrm{OO}}^{-2}\right\rangle^{-1 / 2}-R_{\mathrm{OO}, e}=0.119 \mathrm{bohr}$. This value subtracted from the experimental $\left\langle R_{\mathrm{OO}}^{-2}\right\rangle^{-1 / 2}$ gives $5.505 \mathrm{bohr}$ as the new “experimental" equilibrium separation $R_{\mathrm{OO}, e}$, in excellent agreement with the calculation of Klopper et al. ${ }^{38}$ Use of SAPT-5st gives a slightly smaller value of 5.491 bohr. Thus, we recommend $5.50 \pm 0.01$ bohr as the best current experimental/theoretical estimate of $R_{\mathrm{OO}, e}$. All the SAPT $\mathrm{O}-\mathrm{O}$ distances were computed from center of mass separations using the SAPT-5s monomer orientation from Table X.

Although an excellent agreement between theory and experiment has been reached, one should point out that the compared values of $R_{\mathrm{OO}, e}$ do not represent exactly the same quantity due to different treatments of monomer's geometry. The experimental value of $\left\langle R_{\mathrm{OO}}^{-2}\right\rangle^{-1 / 2}$ was obtained from measured rotational constants by assuming rigid water monomers at their experimental equilibrium geometry. The SAPT-5s potential was also obtained for rigid monomers but at a different, $\langle r\rangle_{0}$, geometry (this, however, must have had a very small effect on $\Delta R_{\mathrm{OO}}$ ). In contrast, calculations of Ref. 38 partially optimized the internal monomer coordinates. One of these coordinates, the donor $\mathrm{O}-\mathrm{H}$ bond length, is increased by 0.012 bohr, while the others are virtually unchanged. This is a rather small effect but comparable to the error estimates of the discussed quantities. One may point out in this connection that the extraction of the "experimental" equilibrium $\mathrm{O}-\mathrm{O}$ distance is inherently not a welldefined problem.

Table $\mathrm{X}$ shows that the SAPT-5s equilibrium $\mathrm{O}-\mathrm{O}$ separation is about 0.08 bohr too large. One reason is, of course, that the basis set and the level of theory in SAPT $a b$ inito calculations is lower than in the calculations of Ref. 38 (and no extrapolation is used). The other reason is our implementation of the $\langle r\rangle_{0}$ monomer geometry which has the $\mathrm{O}-\mathrm{H}$ distance 0.026 bohr larger than the equilibrium distance, ${ }^{24}$ leading naturally to a larger $R_{\mathrm{OO}}$ in the dimer. The $\langle r\rangle_{0}$ monomer geometry provides a better representation of the monomer in the ground vibrational state than the $r_{e}$ geometry. ${ }^{25}$ This is confirmed in the case of water by the highly accurate dimer spectra including the excellent SAPT-5s prediction of the $B+C$ rotational constant (to within $0.5 \%$ of experiment). ${ }^{22}$

\section{Potential depth}

The best current estimate of the global depth at the minimum, $D_{e}$, is the value of $5.02 \pm 0.05 \mathrm{kcal} / \mathrm{mol}(1756 \pm 17$ $\mathrm{cm}^{-1}$ ) from the recent work of Klopper et al. ${ }^{38}$ This estimate is partly based on the two MBPT2 results quoted in Table X: by Schütz et al. ${ }^{34}$ in an extremely large basis set of 1046 functions and by Klopper and Lüthi ${ }^{36}$ in an explicitly correlated basis with the orbital part including 444 functions. For comparison, our basis consists of 112 functions at the dimercentered plus basis set ( $\mathrm{DC}^{+} \mathrm{BS}$ ) level. ${ }^{20}$ The increase of $D_{e}$ achieved in Ref. 38 compared to Refs. 34 and 36 results from the relaxation of the monomer's geometry-which contributes $0.06 \mathrm{kcal} / \mathrm{mol}$ - and from the use of the $\operatorname{CCSD}(\mathrm{T})$ method— $0.02 \mathrm{kcal} / \mathrm{mol}$.

The SAPT minimum depth of $4.86 \mathrm{kcal} / \mathrm{mol}$ agrees fairly well with the limit value of $5.02 \mathrm{kcal} / \mathrm{mol}$, however, this comparison is not fully meaningful due to the different geometries used to obtain the two results. The $\langle r\rangle_{0}$ monomer geometry used by us increases the magnitude of the interaction energy by about $0.12 \mathrm{kcal} / \mathrm{mol}$ relative to the $r_{e}$ geometry near the dimer minimum. ${ }^{24}$ On the other hand, the result of Ref. 38 includes monomer relaxation, also increasing the magnitude of $D_{e}$. To get a more meaningful comparison we performed a SAPT calculation at the Klopper et al. minimum configuration from Table 6 of Ref. 38, resulting in the interaction energy of $-4.763 \mathrm{kcal} / \mathrm{mol}$ (calculated with respect to monomers in their dimer geometry ${ }^{42}$ ). This should be compared not with $D_{e}$ of Ref. 38 but with what they call "vertical" interaction energy. This value can be obtained from the data in the last row of Table 5 of Ref. 38-using their Eq. (6) with the last term neglected-for the value of the deformation parameter $x=0.83$. The result is $-5.043 \mathrm{kcal} / \mathrm{mol}$, showing that the SAPT basis set incompleteness and theory truncation error amounts to $0.28 \mathrm{kcal} / \mathrm{mol}\left(98 \mathrm{~cm}^{-1}\right)$, slightly more than the earlier estimate of $0.2 \mathrm{kcal} / \mathrm{mol}^{24}$

Table $\mathrm{X}$ includes also an empirical estimate (with a significant theoretical component) of the depth of the minimum equal to $D_{e}=5.44 \pm 0.7 \mathrm{kcal} / \mathrm{mol} .{ }^{40}$ Clearly, experiment is not accurate enough to guide the present-day theory. The theoretical component of this estimate is based on the harmonic approximation of dimer vibrational levels. The harmonic frequencies ${ }^{43}$ used in Ref. 40 differ in some cases by $50 \%$ and more from the current best harmonic values for the water dimer. The frequencies of Ref. 43 give the intermolecular zero-point energy (ZPE) of $2.25 \mathrm{kcal} / \mathrm{mol}$ while SAPT-5s predicts ZPE of $1.81 \mathrm{kcal} / \mathrm{mol}$ (anharmonic value). The use of the latter value brings the empirical estimate to $D_{e}=5.00 \pm 0.7 \mathrm{kcal} / \mathrm{mol}$, in a very good agreement with theory. However, the inaccurate frequencies, and in fact the very use of the harmonic frequencies, introduce also an error in the thermal vibrational energy, influencing the empirical estimate of $D_{e}$. As discussed below, removal of this uncertainty does not appear possible at the present time.

\section{Dissociation energy}

Knowledge of the difference between the SAPT-5s potential and the exact one near the minimum makes it possible to obtain an estimate of the exact dissociation energy $D_{0}$. The value of $D_{0}$ from the dynamic calculations on the SAPT-5s potential ${ }^{22}$ is $1067 \mathrm{~cm}^{-1}$. Since the shape of the SAPT-5s potential well near the ground state level must be very close to the exact shape, as shown by the agreement of the spectra with experiment, a more accurate calculation would result in an approximately vertical shift of the well. This means that the value of $D_{0}$ computed from SAPT will be incremented by the value of the shift, i.e., by the error of the SAPT- 5 s potential. This leads to the estimated exact $D_{0}$ $=1165 \pm 54 \mathrm{~cm}^{-1}$, where the error bars-added in the meansquare way-include Klopper's et al. ${ }^{38}$ estimate of accuracy of $D_{e}\left(17 \mathrm{~cm}^{-1}\right)$, a $1 \%$ error of the computed $D_{0}$ due to the 
use of $6 \mathrm{D}$ dynamics $\left(11 \mathrm{~cm}^{-1}\right.$, see below), and an estimated error of assuming the vertical shift $\left(50 \mathrm{~cm}^{-1}\right)$. The latter is an order of magnitude estimate equal to the difference of ZPE between SAPT-5s and SAPT-5st potentials and is probably rather generous taking into account that the shift itself is $98 \mathrm{~cm}^{-1}$. The best experimental value of $D_{0}$ is $1168 \pm 245$ $\mathrm{cm}^{-1} .{ }^{40}$ Our estimate can also be compared to the estimate of Ref. 38 equal to $1154 \pm 33 \mathrm{~cm}^{-1}$. The latter estimate was obtained by subtracting from $D_{e}$ the intermolecular ZPE and the change in the intramolecular ZPE. The intermonomer ZPE was taken as an average from several literature calculations (including our own with the SAPT-pp potential) and amounted to $643 \mathrm{~cm}^{-1}$. SAPT-5s produces a very close value of $632 \mathrm{~cm}^{-1}$. Using the same formula as in Ref. 38 we arrive at $D_{0}=1756-632-(-42)=1166 \pm 24 \mathrm{~cm}^{-1}$, where $-42 \pm 17 \mathrm{~cm}^{-1}$ is the change of intramolecular ZPE.

It may seem counterintuitive that only one of the two methods of estimating $D_{0}$ made use of the change in the intramolecular ZPE. It has been shown in Ref. 25 that if the monomers are taken in their $\langle r\rangle_{0}$ geometry, the dimer dissociation energy computed with such rigid monomers is very close to the dissociation energy from all-dimensional calculations. For Ar-HF the error of such an approach is only $1 \%$ compared to the $4 \%$ error obtained when the $r_{e}$ monomer is used. Thus, an exact 6D potential for water with $\langle r\rangle_{0}$ geometry monomers should give $D_{0}$ to within $11 \mathrm{~cm}^{-1}$ (or $1 \%$ ) of experiment. One may say that the lowering of the dimer potential in the $\langle r\rangle_{0}$ geometry compared to the $r_{e}$ geometry is effectively accounting for the changes in the internal motion of the monomers. In fact, it has been shown in Ref. 25 that the two energetic changes are equal to each other in the lowest order anharmonic approximation. In the case of water dimer the former effect, equal to $0.12 \mathrm{kcal} / \mathrm{mol}$ or $42 \mathrm{~cm}^{-1}$, is indeed identical to the change of intramolecular ZPE estimated in Ref. 38. In contrast, when $D_{0}$ is estimated starting from the value of the global depth of potential $D_{e}$, very close to the depth of the potential for $r_{e}$ monomers, the change of intramolecular ZPE has to be accounted for explicitly. One may also remark that the very separation of the ZPE into inter- and intra-molecular components used in the approach of Ref. 38 is rigorous only in the harmonic approximation.

\section{Enthalpy of dimerization}

The quantity actually measured in Ref. 40 was not $D_{0}$ but rather the enthalpy of dimerization, $\Delta H(T)$, connected with $D_{0}$ - under assumption of the complete separation of the rotational and vibrational motion - by the formula

$$
-D_{0}=\Delta H(T)-\Delta E_{\text {vib,inter }}^{\prime}(T)-\Delta E_{\text {vib,intra }}^{\prime}(T)+4 R T,
$$

where primes are used to point out that only the temperaturedependent components of vibrational thermal energies are included. The contribution from the intramolecular vibrational thermal energy $\Delta E_{\text {vib,intra }}^{\prime}(T)$ is completely negligible. ${ }^{38}$ The intermolecular vibrational thermal energy is defined in the harmonic approximation as

$$
\Delta E_{\mathrm{vib}, \text { inter }}^{\prime}(T)=\sum_{i=1}^{6} \frac{\omega_{i}}{\exp \left(\omega_{i} / R T\right)-1},
$$

where $\omega_{i}$ are water dimer fundamental excitation energies. The dissociation energy was not explicitly given in Ref. 40 but can be easily found from quantities reported there at $T=$ $373 \mathrm{~K}:-D_{0}=-3.59-2.71+2.96=-3.34 \pm 0.7 \mathrm{kcal} / \mathrm{mol}$ $=-1168 \pm 245 \mathrm{~cm}^{-1}$. The uncertainty of the measured enthalpy is $0.5 \mathrm{kcal} / \mathrm{mol}$ but in accordance with Ref. 40 we have included an additional $0.2 \mathrm{kcal} / \mathrm{mol}$ for the uncertainty of $\Delta E_{\text {vib,inter }}^{\prime}(373 \mathrm{~K})$. This quantity was calculated in Ref. 40 using harmonic frequencies from Ref. 43 which are significantly different from the current best values (as mentioned above, discrepancies are of the order of $50 \%$ and more for some frequencies). The values of $\Delta E_{\text {vib,inter }}^{\prime}(373 \mathrm{~K})$ from recent calculations are up to $0.1 \mathrm{kcal} / \mathrm{mol}$ smaller: $2.61 \mathrm{~cm}^{-1}, 44$ $2.65 \mathrm{~cm}^{-1}$ (Ref. 38) [based on Tschumper et al. CCSD(T) frequencies ${ }^{45}$, and $2.67 \mathrm{~cm}^{-1}{ }^{46}$ The SAPT-5s (SAPT-5st) harmonic frequencies give $2.82(2.72) \mathrm{kcal} / \mathrm{mol}$.

Even if harmonic frequencies were known to within one $\mathrm{cm}^{-1}$, this would not result in a more accurate value of $D_{0}$ due to approximations involved in the derivation of Eqs. (6) and (7). First, the rotational motion is completely separated from the vibrational motion while in an accurate treatment the two are coupled. Next, the harmonic approximation is assumed and the sum over all harmonic levels performed explicitly to give Eq. (7). The harmonic frequencies for water dimer are significantly different from the exact ones. The lowest harmonic frequency of the SAPT-5st potential is $41 \%$ too large while the higher ones are about $30 \%$ too large. Strictly speaking one cannot replace the harmonic frequencies in Eq. (7) by anharmonic ones since the summation leading to this formula is not valid for anharmonic frequencies. If one computes Eq. (7) with anharmonic frequencies anyway, the result is $\Delta E_{\text {vib,inter }}^{\prime}(373 \mathrm{~K})=2.90 \mathrm{kcal} / \mathrm{mol}$. [We have used anharmonic frequencies computed on the SAPT5 st potential for the four lowest levels (averages of the levels splitted by tunneling: $86,112,121,140 \mathrm{~cm}^{-1}$ ) and for the remaining two levels lying higher in the spectrum-for which we did not compute anharmonic frequencies-we used the harmonic ones $\left(370\right.$ and $\left.565 \mathrm{~cm}^{-1}\right)$.] This may indicate that the error estimate of $0.2 \mathrm{kcal} / \mathrm{mol}$ for the rovibrational component of the thermal energy given in Ref. 40 will be valid even for the exact harmonic frequencies.

The theoretical value of $D_{0}$ and Eqs. (6) and (7) can be used to estimate $\Delta H(T)$. With SAPT-5st anharmonic (harmonic) frequencies the result is $\Delta H(373 \mathrm{~K})=-3.27(-3.57)$ $\mathrm{kcal} / \mathrm{mol}$ compared to the measured value of $-3.59 \pm 0.5$ $\mathrm{kcal} / \mathrm{mol} .{ }^{40}$ As it is clear from the discussion given above, it is not possible to give any precise estimate of the accuracy of the theoretical numbers. One may wonder whether it would be possible to compute $\Delta H(T)$ from the nearly exact expression

$$
-D_{0}=\Delta H(T)-\Delta E_{\text {rovib,inter }}^{\prime}(T)+\frac{5}{2} R T
$$

with

$$
\Delta E_{\text {rovib,inter }}^{\prime}(T)=\frac{\sum_{i=1} \Delta E_{i} \exp \left(-\Delta E_{i} / R T\right)}{\sum_{i=0} \exp \left(-\Delta E_{i} / R T\right)},
$$



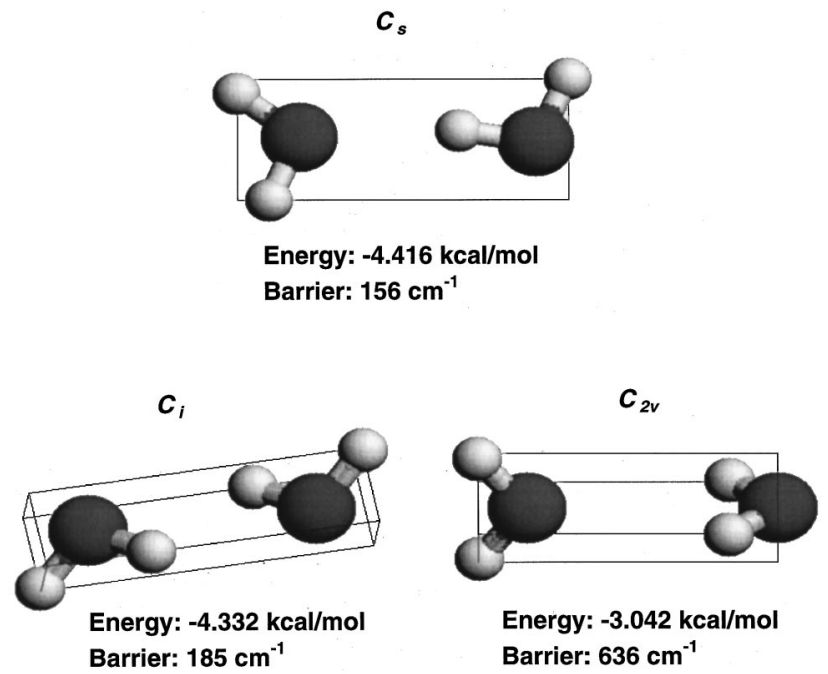

FIG. 6. Saddle points on the SAPT-5s surface.

where the sum is over all intermolecular rovibrational levels (including tunneling splittings) of the water dimer and $\Delta E_{i}$ are the energies of the levels above the ground state energy $\left(\Delta E_{0}=0\right)$. This sum cannot be evaluated at the present since calculations for excited rotational states have not yet been performed except for a few lowest ones. Thus, one would need to assume some kind of separation of the rotational motion. Even then converging the sum over the vibrational part may be difficult since high vibrational excitations are difficult to compute accurately.

\section{B. Saddle points}

Millot et al. ${ }^{8}$ have recently examined characteristic points of 14 water dimer potentials. They have shown that predictions from these potentials are dramatically different from each other as well as from ab initio computed stationary points of Smith et al. ${ }^{47}$ Both Millot et al. and Smith et al. considered not only saddle points of index 1 (number of negative eigenvalues of the Hessian matrix) but also those of higher indexes. The latter do not appear to be particularly relevant for the tunneling processes in the water dimer, therefore we restrict our discussion to points with index 1 . Searches conducted on the SAPT-5s and SAPT-5st potential energy surfaces revealed three such saddle points, labeled by their symmetries $C_{s}, C_{i}$, and $C_{2 v}$. The corresponding geometries are shown in Fig. 6. Since the structures resulting from the SAPT-5st surface are very similar to those from SAPT$5 \mathrm{~s}$, the former ones are not plotted separately. The energies and barriers of the saddle points have been collected in Table $\mathrm{XI}$ and compared to the literature data. Of course, the detailed geometries of the characteristic points listed in the different columns are different between various works. However, the global orientations are the same and in particular spatial symmetry is identical unless otherwise noted.

Table XI includes the results for the VRT(ASP-W) potential of Ref. 23. We were not able to reproduce the minimum parameters published by Fellers et al. ${ }^{23}$ with the potential available on the WWW-site of the Saykally group. None of the VRT(ASP-W) barriers computed by us agrees with the ones published in Table 1 of Ref. 23, the differences ranging from a few to about $20 \mathrm{~cm}^{-1}$. A possible reason for the discrepancies is a larger convergence threshold used in Refs. 23 and 48. In the discussion which follows we will use characteristic points on the VRT(ASP-W) surface computed by us. In addition to the structures reported by Fellers et al. ${ }^{23}$ we have found two additional saddle points on their surface, see Table XI and discussion below.

According to SAPT-5s and SAPT-5st surfaces, the lowest saddle point is the structure $C_{s}$. This structure is particularly important since most likely it lies on the primary pathway of the acceptor tunneling. In the calculations of Smith et $a l .{ }^{47}$ the lowest saddle point was a nonplanar structure $C_{1}$, labeled "structure 2", while $C_{s}$, labeled "structure 3", showed up as a saddle point with index 2 (its index is 1 for all other calculations in Table XI) and the energy higher than

TABLE XI. Energies $(\mathrm{kcal} / \mathrm{mol})$ and barriers $\left(\mathrm{cm}^{-1}\right)$ for the minimum and the saddle points of index 1 . The column "minimum" lists the minimum energies while the other columns list the barriers above the minimum. SAPT-5st is the tuned SAPT-5s and VRT(ASP-W) is the empirical potential of Ref. 23.

\begin{tabular}{lccccc}
\hline \hline & Minimum & $\begin{array}{c}C_{1} \\
\text { acceptor }\end{array}$ & $\begin{array}{c}C_{s} \\
\text { acceptor }\end{array}$ & $\begin{array}{c}C_{i} \\
\text { interchange }\end{array}$ & $\begin{array}{c}C_{2 v} \\
\text { bifurcation }\end{array}$ \\
\hline SAPT-5s & -4.861 & & 156 & 185 & 636 \\
SAPT-5st & -5.029 & & 222 & 248 & 685 \\
VRT(ASP-W) (Ref. 23) $^{\text {VRT(ASP-W) }}$ & -4.91 & 157 & & 207 & 394 \\
& -4.903 & 155 & & 228 & 415 \\
MBPT2 $^{\mathrm{a}}$ & & $161^{\mathrm{b}}$ & & $246^{\mathrm{c}}$ & \\
MBPT4 $^{\mathrm{d}}$ & -5.42 & 206 & $238^{\mathrm{e}}$ & 311 & 682 \\
\hline \hline
\end{tabular}

$\overline{{ }^{a} \text { Computed in the present work using the FORTRAN subroutine from Ref. } 23 \text { (www.cchem.berkeley.edu/ }}$ rjsgrp/).

bonplanar structure of $C_{s}$ symmetry.

"Nonplanar structure of $C_{2}$ symmetry, similar to "structure 5" of Ref. 47

${ }^{\mathrm{d}}$ Ab initio gradient search of Smith et al. (Ref. 47) using 6-311+ $\mathrm{G}(d, p)$ basis and MBPT2 level of theory. CP-uncorrected MBPT2 and MBPT4 energies and barriers listed here used geometries optimized in this way and the $6-311+\mathrm{G}(2 d f, 2 p)$ basis. All calculations of Ref. 47 assumed frozen core approximation.

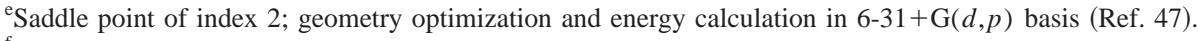

${ }^{\mathrm{f}}$ Saddle point of index 2; calculation at the MBPT2/6-31+G $(d, p)$ optimized geometry in $6-31+\mathrm{G}(2 d, 2 p)$ basis (Ref. 47). 
TABLE XII. Second virial coefficients $\left(\mathrm{cm}^{3} / \mathrm{mol}\right)$. For SAPT-5s the error bars indicate the accuracy of the numerical integration only.

\begin{tabular}{|c|c|c|c|c|c|c|}
\hline \multirow[b]{2}{*}{$T[\mathrm{~K}]$} & \multirow[b]{2}{*}{ Expt. $^{a}$} & \multirow[b]{2}{*}{ Expt. $^{b}$} & \multicolumn{2}{|c|}{ SAPT-pp/ss } & \multicolumn{2}{|c|}{ SAPT-5s } \\
\hline & & & Classical & Total & Classical & Total \\
\hline 273.15 & -1724 & & -2240 & -1880 & $-2039.6 \pm 4.8$ & $-1407.6 \pm 5.9$ \\
\hline 293.15 & -1249 & & -1500 & -1280 & $-1388.9 \pm 3.1$ & $-1029.6 \pm 3.7$ \\
\hline 295.15 & -1211 & & -1440 & -1230 & $-1341.1 \pm 3.0$ & $-1000.2 \pm 3.6$ \\
\hline 298.15 & -1158 & & -1370 & -1172 & $-1273.9 \pm 2.8$ & $-958.2 \pm 3.4$ \\
\hline 323.15 & -816 & & -914 & -797 & $-866.2 \pm 1.9$ & $-690.8 \pm 2.2$ \\
\hline 373.15 & -458 & & -489 & -439 & $-475.9 \pm 1.1$ & $-407.6 \pm 1.2$ \\
\hline 423.15 & -294 & $-275(6)$ & -305 & -279 & $-303.0 \pm 0.7$ & $-270.0 \pm 0.8$ \\
\hline 448.15 & -243 & $-240(5)$ & -250 & -232 & $-250.7 \pm 0.7$ & $-226.4 \pm 0.7$ \\
\hline 473.15 & -206 & $-201(1)$ & -209 & -194 & $-211.1 \pm 0.6$ & $-192.7 \pm 0.6$ \\
\hline 523.15 & -153 & $-150(0.2)$ & -152 & -143 & $-156.0 \pm 0.5$ & $-144.6 \pm 0.5$ \\
\hline 573.15 & -118 & $-116(0.3)$ & -115 & -108 & $-119.9 \pm 0.4$ & $-112.4 \pm 0.4$ \\
\hline 673.15 & -75 & $-73.5(0.2)$ & -71.6 & -68.1 & $-76.5 \pm 0.3$ & $-72.7 \pm 0.3$ \\
\hline 773.15 & -49 & $-49.8(0.2)$ & -47.2 & -43.5 & $-51.9 \pm 0.3$ & $-49.6 \pm 0.3$ \\
\hline 873.15 & -33 & & -32.0 & -30.8 & $-36.4 \pm 0.2$ & $-34.9 \pm 0.2$ \\
\hline 973.15 & -22 & & -21.7 & -20.9 & $-25.7 \pm 0.2$ & $-24.7 \pm 0.2$ \\
\hline
\end{tabular}

${ }^{\mathrm{a}}$ Reference 49

${ }^{\mathrm{b}}$ Reference 50

that of $C_{1}$. Small energy difference between $C_{1}$ and $C_{s}$ and the sensitivity of the index of $C_{s}$ to theory level and basis set indicated the flatness of the potential in this region. In fact, the saddle $C_{1}$ did not appear at all at the Hartree-Fock level of theory. The structure $C_{1}$ has not been detected on SAPT-5s and SAPT-5st surfaces.

Tuning of the SAPT-5s potential (see paper II) resulted in significantly higher barriers. The increases vary from about 30 to $120 \mathrm{~cm}^{-1}$. The increase in the $C_{s}$ barrier heights correlates well with the decrease of the calculated acceptor tunneling splittings upon using SAPT-5st instead of SAPT- $5 \mathrm{~s}{ }^{22}$ This change improved the agreement of SAPT results with experiment.

The $C_{1}$ barrier of the VRT(ASP-W) potential agrees reasonably well with SAPT-5s $C_{s}$ barrier but not with SAPT5st barrier. This is rather surprising since both SAPT-5st and VRT(ASP-W) reproduce the acceptor tunneling splittings very well, being empirically adjusted for this. This shows that heights of barriers themselves are not the only criterion for reaching agreement with experiment. We have found an additional saddle point on the VRT(ASP-W) surface related to acceptor tunneling with energy very close to the $C_{1}$ point. This nonplanar structure has $C_{s}$ symmetry and is similar to the dimer equilibrium structure with increased $\theta$. The MBPT2/MBPT4 $C_{1}$ and $C_{s}$ barriers are much higher than those of VRT(ASP-W) or SAPT-5s but agree quite well with SAPT-5st.

Structure $C_{i}$ is the saddle point on the path of the donoracceptor interchange tunneling which leads to small splittings of the order of $1 \mathrm{~cm}^{-1}$. This barrier has been increased by $63 \mathrm{~cm}^{-1}$ by tuning of SAPT-5s, despite the fact that both potentials produce virtually identical interchange splittings. For this barrier VRT(ASP-W) agrees better with SAPT-5st than with SAPT-5s. Again, we have found an additional saddle point on the VRT(ASP-W) surface having a cyclic nonplanar $C_{2}$ symmetry with energy close to the $C_{i}$ point. The MBPT2/MBPT4 $C_{i}$ barriers are much larger than those of the SAPT and VRT(ASP-W) potentials.
The structure $C_{2 v}$ is on the path of the bifurcation tunneling. This tunneling does not introduce additional splittings of lines. The SAPT-5s barrier was relatively unchanged by tuning and is reasonably close to the MBPT2/MBPT4 barriers. However, the VRT(ASP-W) barriers are lower by more than $200 \mathrm{~cm}^{-1}$.

One should realize that although the differences between the barriers discussed above are significant, the agreement is much better than in the comparison of 14 potentials performed by Millot et al. ${ }^{8}$ where the saddle point energetic structure was qualitatively different between various potentials. Millot $e t$ al. tacitly assumed that the saddle points computed by Smith et al. ${ }^{47}$ were most trustworthy. Since the search for the characteristic points in Ref. 47 was performed in a basis set of lower quality than used in the SAPT calculations (CP-corrected MBPT4 minimum energy of Ref. 47 is $-4.40 \mathrm{kcal} / \mathrm{mol}$ ) and included BSSE, we believe that SAPT-5s saddle points represent the most accurate ab initio predictions.

\section{SECOND VIRIAL COEFFICIENT}

The second virial coefficient for the SAPT-5s potential was calculated with and without the leading order quantum correction and results are compared with experiment and other calculations in Table XII and in Figs. 7 and 8. Figure 7, similar to many literature plots used to compare virial coefficients, shows that the accuracy of current work is so high that differences between various calculations are hardly visible. Therefore, Fig. 8 shows virials in a restricted range of temperatures as differences with respect to the CRC compilation of experimental data. ${ }^{49}$ The expressions for the virial coefficients were taken from Ref. 53 and can also be found in Sec. VI of Ref. 20. The numerical integration was performed by a combination of Monte Carlo (for the angular coordinates) and Stenger quadrature ${ }^{54}$ (for the radial part of the integration). Stenger quadrature was used based on the pre- 


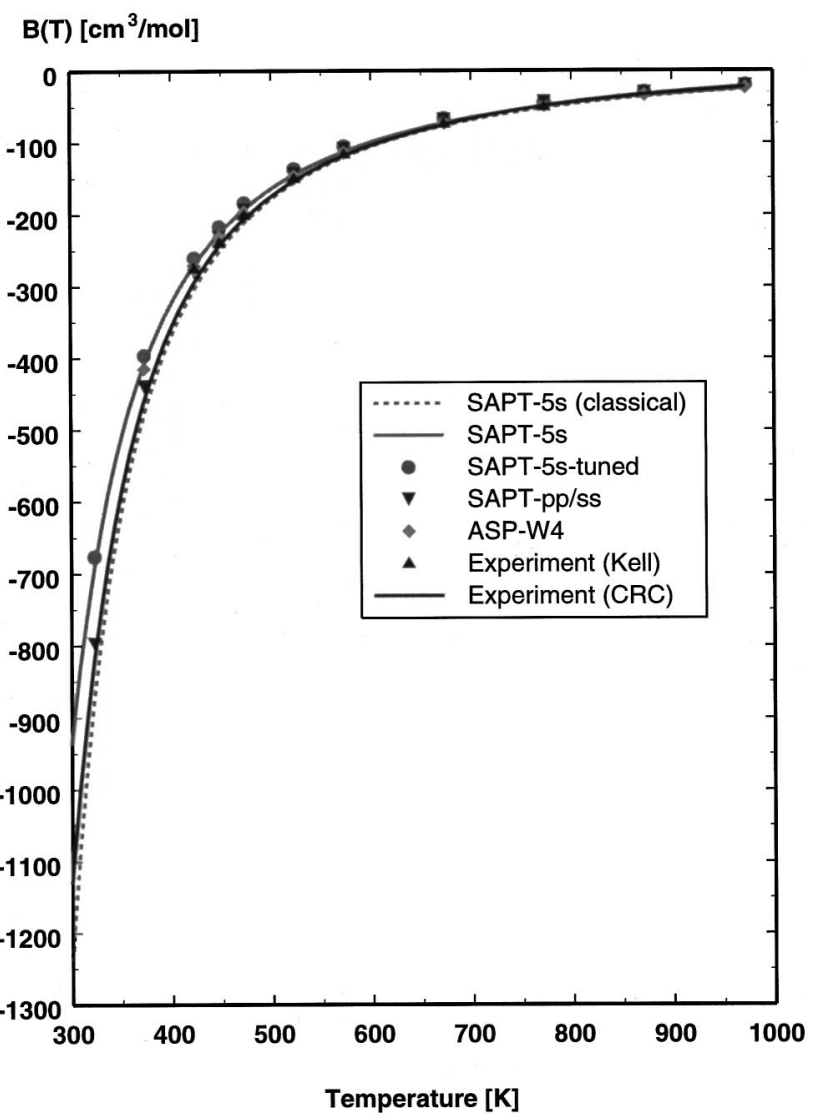

FIG. 7. Comparison of virial coefficients. ASP-W4 is the potential of Ref. 8. Experimental results of Kell et al. are from Ref. 50 while those denoted by CRC are from Ref. 49.

vious experience with second virial coefficient calculations for $\mathrm{CO}_{2}{ }^{55}$ The angular space was sampled by generating 2 million random angular configurations. For each configuration a radial scan was conducted by 15 Stenger quadrature panels each with $2 N+1$ nodes with $N=15$. The first 10 panels were $1 \AA$ long covering distances from 0 to $10 \AA$ and the last 5 were covering: $10-15,15-20,20-30,30-50$, and $50-70 \AA$. The radial integrals are considered to be converged. The errors given in the tables are estimated by the standard deviation

$$
\operatorname{error}(f)=\sqrt{\frac{\left\langle f^{2}\right\rangle-\langle f\rangle^{2}}{N}}
$$

where $N$ is the number of configurations in the angular integration, \langle\rangle is the average over angular configurations, and $f$ stands for the radial integral of the appropriate function, calculated for a fixed set of angular coordinates.

Results of Table XII show that for high temperatures the classical virial coefficients computed using SAPT-5s agree with those obtained with SAPT-pp ${ }^{20}$ to within $15 \%$, with SAPT-5s below SAPT-pp. The two curves cross around 450 $\mathrm{K}$ and at lower temperatures the two virials again become progressively more apart, with the SAPT-5s values above the SAPT-pp values. The difference at the lowest temperature considered amounts to $10 \%$, indicating the larger well volume of SAPT-pp, consistent with the findings discussed above that SAPT-pp was too deep in the small- $R$ part of the potential well.

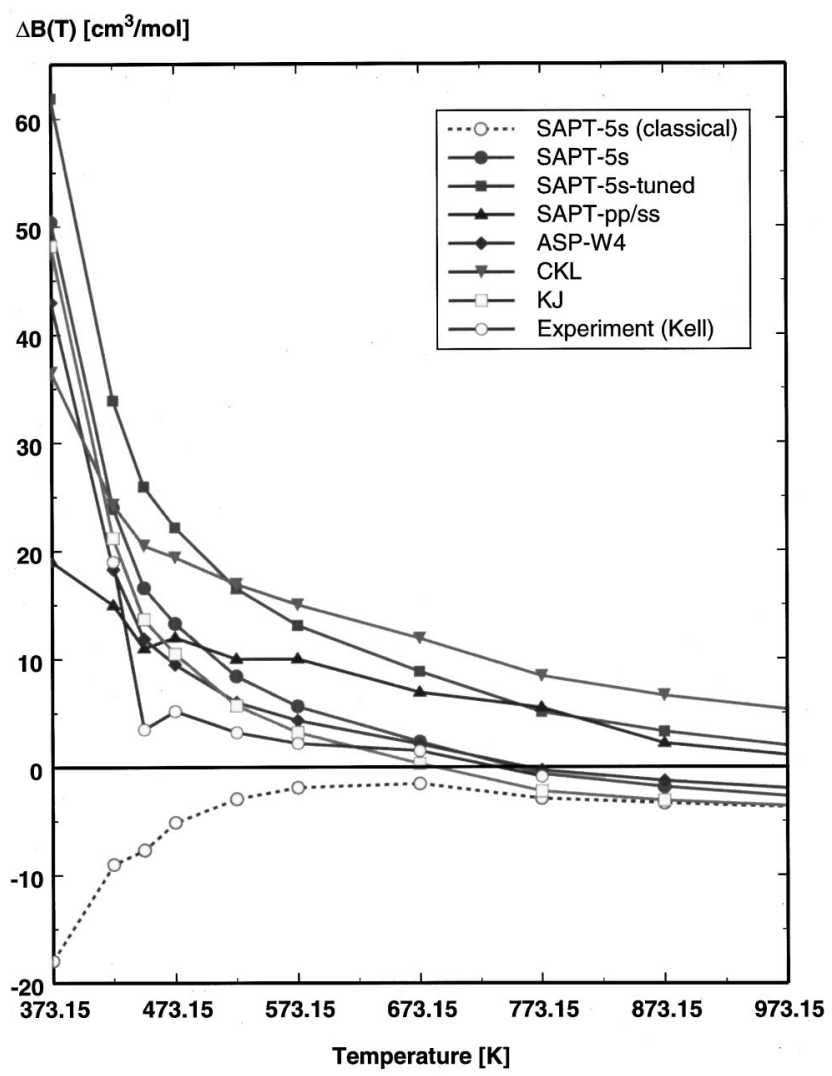

FIG. 8. Comparison of differences in virial coefficients with respect to CRC compilation of experimental results (Ref. 49). The coefficients denoted by CKL and KJ are computed from empirical potentials of Refs. 51 and 52, respectively. See Fig. 7 for other notation.

Differences compared to the results of Ref. 20 are also seen in the quantum part of the virial coefficient. This component was calculated in Ref. 20 using the less accurate SAPT-ss PES. Table XII and Table IV in Ref. 20 show that the values of quantum corrections computed using SAPT- $5 \mathrm{~s}$ are almost a factor of 2 larger at low temperatures. Thus, the quantum correction is very sensitive to the form of the potential. It is also significantly larger than one might expect, amounting at low temperatures to about $30 \%$ of the classical value and dropping below $10 \%$ only for temperatures greater than $200{ }^{\circ} \mathrm{C}$. Thus, any comparison of virial coefficients with experiment should include this term. In the past, calculations of the quantum corrections were rather rare. The most accurate literature calculations are the recent ones by Millot et al. ${ }^{8}$ using the ASP-W, ASP-W2, and ASP-W4 potentials. At $100{ }^{\circ} \mathrm{C}$, the lowest temperature reported in Ref. 8, the quantum correction computed with these potentials varies between 66 and $90 \mathrm{~cm}^{3} / \mathrm{mol}$, the lower value being in good agreement with SAPT-5s. At higher temperatures the agreement is still better, SAPT-5s values being very close to those computed with ASP-W. ${ }^{8}$

Figures 7 and 8 show that SAPT-5s total values of the second virial coefficient lie above experiment for all temperatures, except for $T>700 \mathrm{~K}$. At the highest temperatures the agreement is almost to within the experimental error bars, but at lower temperatures the discrepancies are quite substantial. At the lowest temperature the disagreement reaches nearly $20 \%$. However, these temperatures are in the 
region of supercooled vapor where experimental values are considered less reliable. In fact, Sato et al. ${ }^{56}$ point out that sorption effects dominate over nonideality effects for temperatures below $425 \mathrm{~K}$. At the boiling temperature the difference between the SAPT-5s and experimental virials amounts to $11 \%$. At low temperatures the agreement of SAPT-5s virials with experiment is worse than that for the SAPT-pp/SAPT-ss combination ( $4 \%$ error at $100{ }^{\circ} \mathrm{C}$ ) presented in Ref. 20. Since the SAPT calculations underestimate the depth of the potential by about $0.3 \mathrm{kcal} / \mathrm{mol}$ and since the low temperature virial coefficients are essentially a weighted measure of the volume of the well, our virial coefficients should (and do in the case of SAPT-5s) lie above the experimental values. The better agreement achieved in Ref. 20 should therefore be viewed as somewhat fortuitous, resulting from two factors: the greater depth of SAPT-pp than that of SAPT-5s in some regions of the well and the smaller values of the quantum corrections computed with SAPT-ss compared to the SAPT-5s values, both improving agreement with experiment. Although the insufficient depth is probably the main reason for the present discrepancy between theory and experiment, there are other factors which may contribute to it. One of them are the higher-order quantum corrections which may have to be included at low temperatures. Clearly, if the leading quantum term contributes $30 \%$, the next one is likely to be significant. Furthermore, the effects of the nonrigidity of the monomers may contribute a few percent to the difference. While at low temperatures the present virial coefficients are further from experiment than the values of Ref. 20 , they agree better for high temperatures where experiment is more reliable. SAPT-5s virials are among the best $a b$ initio values published. Only the recent ASP-W2 and ASP-W4 potentials are similarly close to experiment.

The comparison to experiment should take into account experimental uncertainties. Unfortunately, the CRC compilation $^{49}$ does not include any error bars (nor does it give the sources of the data). The differences between the $\mathrm{CRC}$ values and presumably the most accurate measurement by Kell et al. ${ }^{50}$ are much larger than the error bars of the latter work. These differences near $400-500 \mathrm{~K}$ are of the order of $10 \mathrm{~cm}^{3} / \mathrm{mol}$, of similar size as the discrepancies between theory and experiment. For temperatures in the range $600-700 \mathrm{~K}$ the agreement between theory and experiment is excellent, in particular SAPT-5s practically reproduces the Kell et al. ${ }^{50}$ values to within combined experimental uncertainties and theoretical errors of numerical integration (cf. Table XII). At $373 \mathrm{~K}$ Eubank et al. ${ }^{57} \mathrm{ob}-$ tained $451 \pm 18 \mathrm{~cm}^{3} / \mathrm{mol}$, showing the increase of the experimental error bars with the decrease of temperature. For temperatures below $373 \mathrm{~K}$ experimental uncertainties are not available but, as mentioned above, are expected to be large.

Figures 7 and 8 show that SAPT-5st virials agree with experiment somewhat less well than those produced by SAPT-5s. Interestingly enough, SAPT-5st virials lie above those of SAPT-5s despite the fact that at the minimum SAPT-5st potential is $59 \mathrm{~cm}^{-1}$ deeper than SAPT-5s. Clearly, the deepening of the well was accompanied by its narrowing. This is consistent with the finding that $D_{0}$ for SAPT-5st is only $10 \mathrm{~cm}^{-1}$ larger than for SAPT-5s.
TABLE XIII. Change in the second virial coefficient of water when hydrogen is replaced by deuterium. All temperatures are in $\mathrm{K}$ and all coefficients in $\mathrm{cm}^{3} / \mathrm{mol}$.

\begin{tabular}{ccccc}
\hline \hline Temperature & Experiment $^{\mathrm{a}}$ & SAPT-5s & SAPT-ss & MCY $^{\mathrm{b}}$ \\
\hline 423.15 & $15 \pm 7$ & 15 & 11 & 46.5 \\
448.15 & $10 \pm 5$ & 11 & 8.7 & 35.3 \\
473.15 & $3.9 \pm 1$ & 8.3 & 6.7 & 27.6 \\
523.15 & $1.8 \pm 0.3$ & 5.1 & 4.3 & 18.3 \\
573.15 & $0.5 \pm 0.4$ & 3.4 & 2.9 & 13.1 \\
673.15 & $0.6 \pm 0.3$ & 1.7 & 1.6 & 8.01 \\
773.15 & $0.0 \pm 0.2$ & 1.0 & 0.95 & 5.66 \\
\hline \hline
\end{tabular}

${ }^{\text {aReference } 50 .}$

${ }^{\mathrm{b}}$ Reference 58

Figure 8 includes results from empirical polarizable potentials by Cieplak et al. ${ }^{51}$ and by Jordan and Kozack. ${ }^{52}$ It is not surprising that these virials compare well with experiment since virials were used in fitting the potentials. In contrast, most empirical potentials do not use virials in the fitting process since these cannot be fitted well simultaneously with other properties. Virials from some popular empirical potentials displayed in Ref. 20 typically deviate from experiment by a factor of 2 or more. Potentials including polarization effects perform usually better than pure two-body effective potentials but much worse than any of the potentials in Fig. 8 , unless virials are used in fits. It is interesting that the use of virials in the fits gives empirical potentials which do not reproduce the experimental pair distribution functions well, at least this is the case with the potentials of Refs. 51 and 52.

While it is now apparent that the SAPT-ss potential underestimates the quantum correction, this potential was seen to predict quite accurately the isotope shift in the virial coefficient when $\mathrm{H}_{2} \mathrm{O}$ is replaced by $\mathrm{D}_{2} \mathrm{O} .{ }^{20}$ This effect was calculated as the difference of the quantum corrections since the classical part does not depend on the mass, and therefore will be the same for $\mathrm{H}_{2} \mathrm{O}$ and $\mathrm{D}_{2} \mathrm{O}$. The numerical integrations were performed in the same way as for the classical virial coefficient, including 2 million angular configurations. Table XIII shows this shift computed with SAPT-ss, SAPT$5 \mathrm{~s}$, and MCY potentials and compares them to experimental data. One can see that even though the SAPT-5s quantum corrections are considerably larger than the SAPT-ss values, the shift upon changing the hydrogen isotope is virtually left unchanged, preserving the good agreement of this observable with experiment. SAPT-5s shifts are improving the agreement with experiment for the two lowest temperatures, while for higher temperatures SAPT-ss shifts are somewhat closer to experiment.

\section{CONCLUSIONS}

A new pair potential for water, SAPT- $5 \mathrm{~s}$, has been created based on SAPT ab initio calculations. SAPT-5s is a better representation to the ab initio surface than the previous SAPT-pp, mainly because it has been fitted to 2510 grid points while SAPT-pp was fitted to only 1003 . The site-site functional form of SAPT-5s, although considerably simpler than that of SAPT-pp, proved to be equally capable to reproduce the $a b$ initio points. Thanks to its simpler form, SAPT-5s is much faster to calculate than SAPT-pp, which 
enables molecular simulations to be conducted. Overall, SAPT-5s predicts dimer tunneling splitting spectra in excellent agreement with experiment, much better than any previously published potential except for the VRT(ASP-W) potential of Fellers et $_{\text {al. }}{ }^{23}$ which, however, was empirically fitted to these spectra. For the water trimer SAPT-5s combined with a three-body nonadditive component reproduces the spectra much better than VRT(ASP-W) combined with the same three-body part. $^{21}$ The SAPT-5s prediction of the second virial coefficient is slightly inferior compared to the SAPT-pp/SAPT-ss combination for low temperatures but better at higher temperatures where experimental data are more reliable. SAPT-5s predicts the minimum geometry orientation of the dimer in very good agreement with experiment. By the use of SAPT-5s average intermolecular separations in the two lowest rovibrational states, a new empirical value of the equilibrium separation $R_{\mathrm{OO}, e}$ equal to $5.50 \pm 0.01$ bohr is obtained, significantly shorter than the previously accepted result. The minimum distance of SAPT-5s is 0.08 bohr too large compared to this value which is partly due to the $\langle r\rangle_{0}$ monomer geometry used. The minimum depth of SAPT- $5 \mathrm{~s}$ is estimated by comparison with the limit energy from Ref. 38 to be $0.28 \mathrm{kcal} / \mathrm{mol}$ too small. Using the SAPT-5s intermolecular ZPE we obtained a corrected empirical value of $D_{e}=5.00 \pm 0.7 \mathrm{kcal} / \mathrm{mol}$, in very good agreement with the $a b$ initio extrapolated value of Klopper et al. ${ }^{38}$ equal to $5.02 \pm 0.05 \mathrm{kcal} / \mathrm{mol}$. An ab initio estimated limit of the dissociation energy based on SAPT-5s results is $1165 \pm 54 \mathrm{~cm}^{-1}$ compared to the empirical estimate of 1168 $\pm 245 \mathrm{~cm}^{-1} .^{40}$ In summary, we believe that the SAPT-5s PES provides the most accurate representation of the water dimer potential to date.

\section{ACKNOWLEDGMENTS}

We are grateful to Wim Klopper for a discussion of saddle points. This research was supported by NSF Grant No. CHE-9982134 and by an ARO AASERT grant. We acknowledge the use of computing resources made available through the NSF Major Research Instrumentation Program, under Grant No. CTS-9724404, and by the University of Delaware.

${ }^{1}$ M. W. Mahoney and W. L. Jorgensen, J. Chem. Phys. 112, 8910 (2000). ${ }^{2}$ O. Matsuoka, E. Clementi, and M. Yoshimine, J. Chem. Phys. 64, 1351 (1976).

${ }^{3}$ E. Clementi and P. Habitz, J. Phys. Chem. 87, 2815 (1983).

${ }^{4}$ V. Carravetta and E. Clementi, J. Phys. Chem. 81, 2646 (1984).

${ }^{5}$ U. Niessar, G. Corongiu, M.-J. Huang, M. Dupuis, and E. Clementi, Int. J. Quantum Chem., Symp. 23, 421 (1989); U. Niessar, G. Corongiu, E. Clementi, G. R. Kneller, and D. K. Bhattacharya, J. Phys. Chem. 94, 7949 (1990).

${ }^{6}$ G. Corongiu and E. Clementi, J. Chem. Phys. 97, 2030 (1992); 97, 8818 (1992).

${ }^{7}$ C. Millot and A. J. Stone, Mol. Phys. 77, 439 (1992).

${ }^{8}$ C. Millot, J. C. Soetens, M. T. C. M. Costa, M. P. Hodges, and A. J. Stone, J. Phys. Chem. 102, 754 (1998).

${ }^{9}$ I. C. Hayes and A. J. Stone, Mol. Phys. 53, 69 (1984); 53, 83 (1984).

${ }^{10}$ W. Rijks and P. E. S. Wormer, J. Chem. Phys. 90, 6507 (1989); 92, 5754 (1990).

${ }^{11}$ K. T. Tang and J. P. Toennies, J. Chem. Phys. 80, 3726 (1984).

${ }^{12}$ M. M. Szczesniak, R. J. Brenstein, S. M. Cybulski, and S. Scheiner, J. Phys. Chem. 94, 1781 (1990).
${ }^{13}$ K. Szalewicz and B. Jeziorski, Mol. Phys. 38, 191 (1979).

${ }^{14}$ S. Rybak, B. Jeziorski, and K. Szalewicz, J. Chem. Phys. 95, 6576 (1991).

${ }^{15}$ R. Moszynski, B. Jeziorski, A. Ratkiewicz, and S. Rybak, J. Chem. Phys. 99, 8856 (1993)

${ }^{16}$ R. Moszynski, B. Jeziorski, and K. Szalewicz, J. Chem. Phys. 100, 1312 (1994).

${ }^{17}$ R. Moszynski et al., J. Chem. Phys. 100, 5080 (1994).

${ }^{18} \mathrm{~K}$. Szalewicz and B. Jeziorski, in Molecular Interactions_From van der Waals to Strongly Bound Complexes, edited by S. Scheiner (Wiley, New York, 1997), p. 3.

${ }^{19}$ B. Jeziorski and K. Szalewicz, in Encyclopedia of Computational Chemistry, edited by P. v Schleyer et al., (Wiley, Chichester, UK, 1998), Vol. 2, p. 1376

${ }^{20}$ E. M. Mas, K. Szalewicz, R. Bukowski, and B. Jeziorski, J. Chem. Phys. 107, 4207 (1997).

${ }^{21}$ G. Groenenboom et al., Phys. Rev. Lett. 84, 4072 (2000).

${ }^{22}$ G. Groenenboom et al., J. Chem. Phys. 113, 6702 (2000), following paper.

${ }^{23}$ R. S. Feller, et al., Science 284, 945 (1999).

${ }^{24}$ E. M. Mas and K. Szalewicz, J. Chem. Phys. 104, 7606 (1996).

${ }^{25}$ M. Jeziorska, P. Jankowski, K. Szalewicz, and B. Jeziorski, J. Chem. Phys. 113, 2957 (2000).

${ }^{26}$ P. E. S. Wormer and H. Hettema, J. Chem. Phys. 97, 5592 (1992).

${ }^{27} \mathrm{P}$. E. S. Wormer and $\mathrm{H}$. Hettema, POLCOR package, University of $\mathrm{Ni}-$ jmegen, 1992.

${ }^{28}$ J. L. Prather, Energy Levels in Crystals, Natl. Bureau of Standards Monograph 19 (National Bureau of Standards, Washington, DC, 1961).

${ }^{29}$ A. J. Stone, The Theory of Intermolecular Forces (Clarendon, Oxford, 1996).

${ }^{30}$ S. A. Clough, Y. Beers, G. P. Klein, and L. S. Rothman, J. Chem. Phys. 59, 2254 (1973); T. R. Dyke and J. S. Muenter ibid. 59, 3125 (1973).

${ }^{31}$ J. Verhoeven and A. Dymanus, J. Chem. Phys. 52, 3222 (1970).

${ }^{32}$ A FORTRAN subroutine calculating the SAPT- 5 s and SAPT-5st potentials is available as AIP Document No. EPAPS: E-PRLTAO-84-060018. This document may be retrieved via the EPAPS homepage (http://www.aip.org/ pubservs/epaps.html) or from ftp.aip.org in the directory /epaps/. See the EPAPS homepage for more information.

${ }^{33}$ A. Misquitta, R. Bukowski, and K. Szalewicz, J. Chem. Phys. 112, 5308 (2000).

${ }^{34}$ M. Schütz et al., J. Chem. Phys. 107, 4597 (1997).

${ }^{35}$ A. Halkier et al., Theor. Chem. Acc. 97, 150 (1997).

${ }^{36}$ W. Klopper and H. P. Lüthi, Mol. Phys. 96, 559 (1999).

${ }^{37}$ J. G. C. M. van Duijneveldt-van de Rijdt and F. B. van Duijneveldt, J. Chem. Phys. 111, 3812 (1999).

${ }^{38}$ W. Klopper, J. G. C. M. van Duijneveldt-van de Rijdt, and F. B. van Duijneveldt, Phys. Chem. Chem. Phys. 2, 2227 (2000).

${ }^{39}$ J. A. Odutola and T. R. Dyke, J. Chem. Phys. 72, 5062 (1980).

${ }^{40}$ L. A. Curtiss, D. J. Frurip, and M. Blander, J. Chem. Phys. 71, 2703 (1979).

${ }^{41}$ S. F. Boys and F. Bernardi, Mol. Phys. 19, 553 (1970).

${ }^{42}$ K. Szalewicz and B. Jeziorski, J. Chem. Phys. 109, 1198 (1998).

${ }^{43}$ L. A. Curtiss and J. A. Pople, J. Mol. Spectrosc. 55, 1 (1975).

${ }^{44}$ M. J. Frisch, J. E. Del Bene, J. S. Binkley, and H. F. Schaefer III, J. Chem. Phys. 84, 2279 (1986).

${ }^{45}$ G. S. Tschumper, M. L. Leininger, B. C. Hoffman, and H. F. Schaefer III (unpublished).

${ }^{46}$ S. S. Xantheas and T. H. Dunning, Jr., J. Chem. Phys. 99, 8774 (1993).

${ }^{47}$ B. J. Smith, D. J. Swanton, J. A. Pople, and H. F. Schaefer III, J. Chem. Phys. 92, 1240 (1990).

${ }^{48}$ R. S. Fellers (private communication).

${ }^{49}$ CRC Handbook of Chemistry and Physics, edited by D. R. Lide (CRC, Boca Raton, New York, London, Tokyo, 1995), pp. 6-30, 6-31.

${ }^{50}$ G. S. Kell, G. E. McLaurin, and E. Whalley, Proc. R. Soc. London, Ser. A 425, 49 (1989).

${ }^{51}$ P. Cieplak, P. Kollman, and T. Lybrand, J. Chem. Phys. 92, 6755 (1990).

${ }^{52}$ R. E. Kozack and P. C. Jordan, J. Chem. Phys. 96, 3120 (1992).

${ }^{53}$ C. G. Gray and K. E. Gubbins, Theory of Molecular Fluids Volume 1: Fundamentals (Clarendon, Oxford, 1984).

${ }^{54}$ F. Stenger, SIAM Rev. 23, 165 (1981).

${ }^{55}$ R. Bukowski et al., J. Chem. Phys. 110, 3785 (1999).

${ }^{56}$ H. Sato et al., J. Phys. Chem. Ref. Data 20, 1023 (1991).

${ }^{57}$ P. T. Eubank, L. L. Joffrion, M. R. Patel, and W. Warowny, J. Chem. Thermodyn. 20, 1009 (1988).

${ }^{58}$ Z. Slanina, J. Chem. Phys. 142, 525 (1990). 